\title{
Real Lie Algebras of Differential Operators and Quasi-Exactly Solvable Potentials
}

\author{
Artemio González-López ${ }^{\dagger}$ \\ Departamento de Física Teórica II \\ Universidad Complutense \\ 28040 Madrid \\ SPAIN \\ artemio@ciruelo.fis.ucm.es
}

\author{
Niky Kamran * \\ Department of Mathematics \\ McGill University \\ Montréal, Québec \\ CANADA H3A $2 \mathrm{~K} 6$ \\ nkamran@math.mcgill.ca
}

\author{
Peter J. Olver ${ }^{\S}$ \\ School of Mathematics \\ University of Minnesota \\ Minneapolis, MN 55455 \\ olver@ima . umn .edu
}

\begin{abstract}
We first establish some general results connecting real and complex Lie algebras of first-order differential operators. These are applied to completely classify all finite-dimensional real Lie algebras of first-order differential operators in $\mathbb{R}^{2}$. Furthermore, we find all algebras which are quasi-exactly solvable, along with the associated finitedimensional modules of analytic functions. The resulting real Lie algebras are used to construct new quasi-exactly solvable Schrödinger operators on $\mathbb{R}^{2}$.
\end{abstract}

$\dagger$ Supported in part by DGICYT Grant PB92-0197.

* Supported in part by an NSERC Grant.

$\S$ Supported in part by NSF Grants DMS 92-04192 and 95-00931.

November 7, 1995 


\section{Introduction.}

Over the past decade, a great deal of effort has been directed towards the construction of physically significant quantum mechanical systems for which the spectral problem associated to the Schrödinger operator may not be exactly solvable, but for which at least part of the spectrum can be computed exactly by algebraic methods. The concept of a "spectrum generating algebra" appears to date back to a 1959 paper of Goshen and Lipkin, [15], which seems to have remained largely unnoticed. Spectrum-generating algebras were independently rediscovered in 1965 by two groups of theoretical physicists, $[\mathbf{3}],[\mathbf{6}]$. This served as the impetus for a great deal of subsequent research activity, as evidenced by the two volume set of reprints [5], and the more recent conference proceedings [16]. A survey of the history and contributions appears in the review paper of Bohm and Ne'eman, [4], at the start of these volumes. Applications of spectrum generating algebras to molecular spectroscopy began in the early 1980's with the work of Iachello, Levine, Alhassid, Gürsey, and collaborators; see the recent book, $[\mathbf{1 8}]$, for a survey of the theory and applications. In both the nuclear and spectroscopic applications, the relevant Hamiltonian is a Liealgebraic differential operator, meaning that it lies in the universal enveloping algebra of a finite-dimensional Lie algebra of first-order differential operators — the "hidden symmetry algebra" of the quantum mechanical problem.

In the late 1980's, Shifman, Turbiner, Ushveridze, and their collaborators undertook the analysis of a significant new class of Schrödinger operators which they called "quasiexactly solvable", $[\mathbf{2 7}],[\mathbf{2 8}],[\mathbf{2 9}]$. A Lie-algebraic differential operator satisfies the additional condition of quasi-exact solvability provided its hidden symmetry algebra admits a finite-dimensional representation space (or module) consisting of smooth functions on the underlying space. Since the differential operator is a polynomial in the first-order operators generating the symmetry algebra, it leaves the associated module invariant, and hence restricts to a finite-dimensional linear transformation thereon. If the functions in the module are square-integrable with respect to the natural measure, the quasi-exactly solvable operator is said to be normalizable; in this case, the eigenvalues of the restricted linear transformation will correspond to part of the point spectrum of the differential operator, thereby reducing the computation of that part of the spectrum to an algebraic eigenvalue problem. See $[\mathbf{3 0}]$ for a comprehensive survey of the theory and applications of quasi-exactly solvable systems in physics.

In non-relativistic quantum mechanics one is interested in real-valued Schrödinger operators of the form $H=-\Delta+V$, where $\Delta$ is the Laplacian (kinetic energy) operator on a finite-dimensional real Riemannian manifold ${ }^{\dagger} M$, and $V$ is the potential. (The physical units are taken so that $\hbar=2 m=1$.) Thus, $H$ is quasi-exactly solvable if there exists

$\dagger$ More generally, for multi-particle systems, the kinetic energy operator would be a sum of Laplacians. But such systems fit readily into our general framework, since a sum of Laplacians is just the Laplacian operator on a Cartesian product manifold whose metric tensor is the direct sum of the individual metrics. 
a finite-dimensional Lie algebra $\mathfrak{g}$ of first-order differential operators, admitting a finitedimensional module $\mathfrak{N}$ of smooth functions, such that $H$ can be written as a bilinear combination

$$
H=\sum_{a, b=1}^{r} C_{a b} T^{a} T^{b}+\sum_{a=1}^{r} C_{a} T^{a}
$$

of the generators $T^{a}$ of $\mathfrak{g}$. Here $r=\operatorname{dim} \mathfrak{g}$, the $C_{a b}$ 's and $C_{a}$ 's are real constants, and we have omitted an irrelevant constant term that can be absorbed in the energy. Since the quantum mechanical wave functions are, in general, complex-valued, the module $\mathfrak{N}$ can contain complex-valued functions. Clearly, since each of the generators of $\mathfrak{g}$ leaves $\mathfrak{N}$ invariant, $T^{a}(\mathfrak{N}) \subset \mathfrak{N}$, the Hamiltonian $H$ admits $\mathfrak{N}$ as a finite-dimensional invariant space, $H(\mathfrak{N}) \subset \mathfrak{N}$. Thus, assuming again that the functions in $\mathfrak{N}$ are normalizable, the spectrum of a quasi-exactly solvable Schrödinger operator $H$ has an algebraic sector, which can be computed algebraically by diagonalizing the finite-dimensional linear operator obtained by restricting $H$ to $\mathfrak{N}$. In the decomposition (1), even though $H$ is required to be a real differential operator, the generators $T^{a}$ of the hidden symmetry algebra $\mathfrak{g}$ could conceivably be complex-valued, with complex coefficients $C_{a b}, C_{a}$ also. In practice, however, if the operators $T_{a}$ are complex-valued the conditions on the complex constants $C_{a b}$ and $C_{a}$ arising from the fact that $H$ must be a real differential operator - i.e., the potential $V$ must be a real-valued function - are virtually impossible to satisfy. For instance, extensive symbolic algebra calculations using the realization of $\mathfrak{s o}(3, \mathbb{R})$ given by Case 4.2 in Table 4 failed to yield any real-valued potentials, even after allowing for a possible rescaling of the wave function by a non-zero gauge factor, $\mathrm{cf}$. (7). Therefore, the primary objects of interest for the construction of real quasi-exactly solvable Schrödinger operators are finite-dimensional real Lie algebras of real-valued first-order differential operators on an open subset $M$ of $m$-dimensional Euclidean space, which admit a finite-dimensional module of (smooth) complex-valued wave functions. It is the classification of such objects, particularly in the two-dimensional case, that forms the principal focus of this paper.

One of the principal research goals in this subject has been to obtain and classify new and physically important examples of quasi-exactly solvable Schrödinger operators. In the one-dimensional case, complete results are known. In this case, the real and complex classifications are identical, since, up to equivalence, there is essentially just one family of one-dimensional quasi-exactly solvable Lie algebras of first-order differential operators, indexed by a single quantum number $n \in \mathbb{N}$. The symmetry algebra can be identified with the unimodular Lie algebra $\mathfrak{s l}(2, \mathbb{C})$ corresponding to the projective group action, having its standard representation on the space of polynomials of degree at most $n$. The complete list of one-dimensional quasi-exactly solvable Schrödinger operators was found in [28]; further, a complete solution to the normalizability problem for these operators was recently determined, $[\mathbf{1 2}],[\mathbf{1 4}]$. The higher-dimensional case is much more challenging, owing notably to the fact that, already in the case of planar vector fields, there are infinitely many distinct finite-dimensional Lie algebras of vector fields, of arbitrarily large dimension. Moreover, some of the complex Lie algebras have several different inequivalent real forms, and so the classification of complex quasi-exactly solvable Lie algebras or differential operators does not fully resolve the corresponding real problem. In the two-dimensional case, a complete list of Lie algebras of first-order differential operators in two complex 
variables was found by us in $[\mathbf{9}],[\mathbf{1 0}]$. Our starting point was Lie's complete classification of the finite-dimensional Lie algebras of vector fields in two complex variables, [20], [21], and then applying methods based on Lie algebra cohomology to determine the associated Lie algebras of differential operators. In [13], this classification was applied to construct several new families of normalizable quasi-exactly solvable Schrödinger operators in two dimensions, on both flat and curved spaces. The complete classification of (normalizable) quasi-exactly solvable Schrödinger operators remains to be done, although this appears to be an extremely difficult problem. In this paper, we complete the local classification of Lie algebras of first-order differential operators in two real variables, using as our starting point the classification of Lie algebras of vector fields in $\mathbb{R}^{2}$ that was rigorously established in [11]. Interestingly, for the five additional real forms not appearing in Lie's complex classification, every associated Lie algebra of differential operators is a subalgebra of a discrete family of algebras isomorphic to the pseudo-orthogonal Lie algebra $\mathfrak{s o}(3,1)$ - the linear isometries of Minkowski space. Our classification will be applied to find a few interesting new examples of real quasi-exactly solvable Schrödinger operators in two-dimensional space.

As for the extension of these results to more than two dimensions, the difficulty is that there is still no complete classification of even the Lie algebras of vector fields in $\mathbb{C}^{3}$. Lie, $[\mathbf{2 0}]$, describes a substantial fraction of the classification, and indicates that he had completed it, although, as far as we know, this has never appeared in print! Nevertheless, it would be an interesting and physically relevant problem to extend the classification even to those examples discussed by Lie. Our methods for passing from a complex Lie algebra of differential operators to its associated real forms and the quasi-exact solvability conditions are of general applicability, and can be readily applied to higher dimensional situations for which one can determine relevant real forms.

The paper is organized as follows. In Section 2, we review the basic theory of Lie algebras of differential operators, describing their characterization based on a Lie algebra of vector fields, an associated module or representation space, and a cohomology class in the associated Lie algebra cohomology. In Section 3 we describe the basic connection between complex Lie algebras of differential operators and their real-analytic counterparts. The basic result that allows us to straightforwardly pass from real forms of the Lie algebra of vector fields to corresponding real forms of the Lie algebra of differential operators is that any complexified Lie algebra of vector fields with a complexified module automatically has complexified cohomology. In Section 4 we review the basic facts about quasi-exact solvability and the quantization of cohomology in the complex case, and its real-analytic counterparts. In Section 5, we complete the classification of real Lie algebras of differential operators in the plane along with the quasi-exact solvability condition and associated modules. In particular, we show that, of the five additional Lie algebras of vector fields in the real plane not being simple restrictions of the complex ones in Lie's list, the only one admitting an extension to a Lie algebra of first-order differential operators by a non-trivial real-valued cocycle is a family central extension of $\mathfrak{s o}(3,1)$, for which the corresponding Lie algebras of first-order differential operators are labeled by a non-negative integer. As an application, we obtain the explicit decomposition into irreducible submodules of the modules obtained by Morozov et al., $[24]$, for the case of $\mathfrak{s o}(3, \mathbb{R})$. Finally, in Section 6 , we 
review the theory of quasi-exactly solvable Schrödinger operators, and use the preceding results to both reconstruct the family of operators found by Zaslavskii, [31], as well as exhibit new real quasi-exactly solvable potentials in two variables.

\section{Lie Algebras of Differential Operators.}

First, we review the basic theory underlying the classification of Lie algebras of differential operators in the complex domain, referring the reader to [14] for details. Let $M$ be a complex-analytic manifold ${ }^{\dagger}$ of dimension $m$. Let $\mathcal{F}(M)=\mathcal{F}(M, \mathbb{C})$ denote the space of complex-valued analytic functions on $M$. Let $\mathcal{V}(M)$ denote the space of analytic vector fields on $M$, which forms an infinite-dimensional Lie algebra based on the standard Lie bracket operation $[\mathbf{v}, \mathbf{w}]$. The space $\mathcal{F}(M)$ forms a module, or representation space, for the Lie algebra $\mathcal{V}(M)$, which acts via derivations: $h \mapsto \mathbf{v}(h)$ for $\mathbf{v} \in \mathcal{V}(M), h \in \mathcal{F}(M)$. Similarly, $\mathcal{F}(M)$ acts on itself by multiplication, i.e., a function $f \in \mathcal{F}(M)$ defines the linear multiplication operator $h \mapsto f \cdot h$ for $h \in \mathcal{F}(M)$. The Lie algebra of first-order differential operators on $M$ can be identified with the semidirect product of these two Lie algebras, $\mathcal{D}^{1}(M)=\mathcal{V}(M) \ltimes \mathcal{F}(M)$, so that each element $T \in \mathcal{D}^{1}(M)$ decomposes into a sum $T=\mathbf{v}+f$ of a vector field $\mathbf{v}$ and a multiplication operator $f$. Again, $\mathcal{F}(M)$ defines a $\mathcal{D}^{1}(M)$-module, with $T(h)=\mathbf{v}(h)+f \cdot h$ for $h \in \mathcal{F}(M)$. The Lie bracket or commutator is then

$[T, U]=T U-U T=[\mathbf{v}, \mathbf{w}]+\mathbf{v}(g)-\mathbf{w}(f), \quad$ where $\quad T=\mathbf{v}+f, \quad U=\mathbf{w}+g \in \mathcal{D}^{1}(M)$.

In local coordinates $z=\left(z^{1}, \ldots, z^{m}\right)$, a first-order differential operator has the form

$$
T=\mathbf{v}+f=\sum_{i=1}^{m} \xi^{i}(z) \frac{\partial}{\partial z^{i}}+f(z)
$$

where the coefficients $\xi^{i}$ and $f$ are analytic functions of $z$. We let $\pi: \mathcal{D}^{1}(M) \rightarrow \mathcal{V}(M)$, with $\pi(\mathbf{v}+f)=\mathbf{v}$, denote the natural projection of a first-order differential operator onto its vector field part. We can identify $\pi(T)=\mathbf{v}$ with the "symbol" of the differential operator $T=\mathbf{v}+f$.

We are interested in studying Lie subalgebras $\mathfrak{g} \subset \mathcal{D}^{1}(M)$, so that $\mathfrak{g}$ is a Lie algebra of first-order differential operators on the manifold $M$. Our classification of finite-dimensional subalgebras will be local, leaving aside extremely interesting, but less well understood global issues. Since all our results are local, from now on we will usually avoid explicit use of the term "local". By (2), the vector field part, or "symbol", of the algebra, defined as $\mathfrak{h}=\pi(\mathfrak{g}) \subset \mathcal{V}(M)$, forms a Lie algebra of vector fields on $M$. Let $\mathfrak{M}=\mathfrak{g} \cap \mathcal{F}(M)$ denote the subspace of $\mathfrak{g}$ consisting of all the multiplication operators in $\mathfrak{g}$. The commutator formula (2) (with $\mathbf{w}=0$ ) immediately implies that $\mathfrak{M}$ is an $\mathfrak{h}$-module, meaning that if $\mathbf{v} \in \mathfrak{h}$ and $h \in \mathfrak{M}$, then $\mathbf{v}(h) \in \mathfrak{M}$; in other words, $\mathfrak{M}$ forms a representation space for the Lie algebra

$\dagger$ In our applications, $M$ will always be an open subset of Euclidean space, but the basic theory in this section is valid in general. 
$\mathfrak{h}$. If $T=\mathbf{v}+f \in \mathfrak{g}$, it is tempting to define $F: \mathfrak{h} \rightarrow \mathcal{F}(M)$ by $\langle F ; \mathbf{v}\rangle=f$. However, this is incorrect, since we can clearly add to $T$ any function $\widetilde{h} \in \mathfrak{M}$, and still obtain an element of $\mathfrak{g}$ with the same symbol $\mathbf{v}$ as $T$. To remedy this, we just go to the quotient space $\mathcal{Q}=\mathcal{F}(M) / \mathfrak{M}$, which also forms an $\mathfrak{h}$-module, and define $F: \mathfrak{h} \rightarrow \mathcal{Q}$ by

$$
\langle F ; \mathbf{v}\rangle=[f] \in \mathcal{Q}, \quad \text { if } \quad \mathbf{v} \in \mathfrak{h} \quad \text { and } \quad \mathbf{v}+f \in \mathfrak{g}
$$

This is clearly well defined, since if $\mathbf{v} \in \mathfrak{h}$ and $T_{1}=\mathbf{v}+f_{1} \in \mathfrak{g}, T_{2}=\mathbf{v}+f_{2} \in \mathfrak{g}$, then $T_{1}-T_{2}=f_{1}-f_{2} \in \mathfrak{g}$ implies that $f_{1}-f_{2} \in \mathfrak{M}$, and therefore $F(\mathbf{v})=\left[f_{1}\right]=\left[f_{2}\right]$. In view of the commutator formula (2), the fact that the differential operators in $\mathfrak{g}$ form a Lie algebra implies that $F$ must satisfy the bilinear identity

$$
\mathbf{v}\langle F ; \mathbf{w}\rangle-\mathbf{w}\langle F ; \mathbf{v}\rangle-\langle F ;[\mathbf{v}, \mathbf{w}]\rangle=0, \quad \mathbf{v}, \mathbf{w} \in \mathfrak{h} .
$$

In the language of Lie algebra cohomology, $[\mathbf{1 7}]$, the left hand side of (5) defines the differential $\delta F$ of the linear map $F$; the fact that it vanishes implies that $F$ defines a 1 -cocycle on $\mathfrak{h}$ with values in the $\mathfrak{h}$-module $\mathcal{Q}$. Let $Z^{1}(\mathfrak{h}, \mathcal{Q})$ denote the space of $\mathcal{Q}$-valued 1 -cocycles on the Lie algebra $\mathfrak{h}$. The basic classification theorem, $[14]$, can be stated as follows.

Proposition 1. Let $\mathfrak{g} \subset \mathcal{D}^{1}(M)$ be a Lie algebra of first-order differential operators. Then $\mathfrak{g}$ can be represented by a triple $(\mathfrak{h}, \mathfrak{M}, F)$, where:

(i) $\mathfrak{h}=\mathfrak{g} \cap \mathcal{V}(M)$ is a Lie algebra of vector fields on $M$,

(ii) $\mathfrak{M}=\mathfrak{g} \cap \mathcal{F}(M)$ is an $\mathfrak{h}$-module of scalar-valued functions,

(iii) $F \in Z^{1}(\mathfrak{h}, \mathcal{Q})$ is a $\mathcal{Q}=\mathcal{F}(M) / \mathfrak{M}$-valued 1-cocycle on $\mathfrak{h}$.

There are two classes of equivalence maps that preserve the basic Lie algebra structure of the space $\mathcal{D}^{1}(M)$. The first are the changes of variables, provided by local diffeomorphisms $\varphi: M \rightarrow M$, which act naturally on $\mathcal{D}^{1}(M)$ via

$$
\varphi_{*}(T)=\varphi_{*}(\mathbf{v}+f)=d \varphi(\mathbf{v})+f \circ \varphi^{-1}
$$

where $d \varphi$ is the usual differential (push-forward) map on vector fields. The second are the gauge transformations, obtained by multiplying the functions in $\mathcal{F}(M)$ by a fixed non-vanishing function ${ }^{\dagger} \eta(x)=e^{\sigma(x)}$. The corresponding gauge action on a differential operator is given by

$$
\mathcal{G}_{\sigma}(T)=e^{-\sigma} \cdot T \cdot e^{\sigma} \quad \text { so that } \quad \mathcal{G}_{\sigma}(\mathbf{v}+f)=\mathbf{v}+f+\mathbf{v}(\sigma)
$$

Thus, the effect of a gauge transformation is to modify the multiplication part of the differential operator by the additional term $\mathbf{v}(\sigma)$.

$\dagger$ Here, in contrast to the usual physics version, the gauge factor $\eta$ is not assumed to be unitary, i.e., $\sigma$ is not necessarily purely imaginary. 
Definition 2. Two Lie algebras of differential operators $\mathfrak{g}$ and $\tilde{\mathfrak{g}}$ are equivalent if and only if there is an equivalence map $\Psi=\left(\varphi, \mathcal{G}_{\sigma}\right)$, consisting of a change of variables and a gauge transformation, that maps one to the other, so $\tilde{\mathfrak{g}}=\mathcal{G}_{\sigma} \circ \varphi_{*}(\mathfrak{g})$.

If the operators $T$ generate a Lie algebra $\mathfrak{g} \subset \mathcal{D}^{1}(M)$, which we identify with the triple $(\mathfrak{h}, \mathfrak{M}, F)$ as above, then we can interpret the gauge term $\mathbf{v}(\sigma)$ in $(7)$ as the coboundary map $\delta \sigma: \mathfrak{h} \rightarrow \mathcal{Q}$, defined by $\langle\delta \sigma ; \mathbf{v}\rangle=\mathbf{v}(\sigma)$, associated with the 0 -cochain $\sigma \in \mathcal{Q}$ on the Lie algebra $\mathfrak{h}$. Note that for $\sigma \in \mathfrak{M}$ the coboundary $\delta \sigma$ modifies the cocycle $F$ in a trivial manner, since $F$ is only defined modulo elements of $\mathfrak{M}$ anyway. Thus, a gauge transformation $\mathcal{G}_{\sigma}$ will map the Lie algebra $\mathfrak{g}=(\mathfrak{h}, \mathfrak{M}, F)$ to the cohomologous Lie algebra $\mathcal{G}_{\sigma}(\mathfrak{g})=(\mathfrak{h}, \mathfrak{M}, F+\delta \sigma)$. Let $B^{1}(\mathfrak{h}, \mathcal{Q})$ denote the space of $\mathcal{Q}$-valued coboundaries, and $H^{1}(\mathfrak{h}, \mathcal{Q})=Z^{1}(\mathfrak{h}, \mathcal{Q}) / B^{1}(\mathfrak{h}, \mathcal{Q})$ the corresponding first Lie algebra cohomology space. We conclude that the gauge-equivalent Lie algebras corresponding to a given Lie algebra of vector fields $\mathfrak{h}$ and module $\mathfrak{M}$ are classified by cohomology classes $[F] \in H^{1}(\mathfrak{h}, \mathcal{Q})$. In other words, equivalent Lie algebras correspond to equivalent triples $(\mathfrak{h}, \mathfrak{M},[F]$, and $(\widetilde{\mathfrak{h}}, \widetilde{\mathfrak{M}},[\widetilde{F}])$, meaning that there is a change of variables $\varphi$ for which $\widetilde{\mathfrak{h}}=\varphi_{*}(\mathfrak{h}), \widetilde{\mathfrak{M}}=\varphi_{*}(\mathfrak{M})$, and $\widetilde{F}=\varphi_{*} \circ F \circ \varphi_{*}^{-1}+\delta \sigma$. In particular, if $F$ is cohomologous to a coboundary, then the Lie algebra $\mathfrak{g}$ is equivalent, under a gauge transformation, to the sum $\widetilde{\mathfrak{g}}=\widetilde{\mathfrak{h}}+\mathfrak{M}$ of a Lie algebra of pure vector fields and a subspace of multiplication operators.

Theorem 3. There is a one-to-one correspondence between equivalence classes of Lie algebras $\mathfrak{g}$ of first-order differential operators on $M$ and equivalence classes of triples $(\mathfrak{h}, \mathfrak{M},[F])$, where

(i) $\mathfrak{h}$ is a Lie algebra of vector fields,

(ii) $\mathfrak{M} \subset \mathcal{F}(M)$ is an $\mathfrak{h}$-module of functions,

(iii) $[F]$ is a cohomology class in $H^{1}(\mathfrak{h}, \mathcal{Q})$, where $\mathcal{Q}=\mathcal{F}(M) / \mathfrak{M}$.

The classification of Lie algebras of first-order differential operators therefore reduces to the problem of classifying triples $(\mathfrak{h}, \mathfrak{M},[F])$ under local changes of variables. From the standpoint of physics, the most interesting are the finite-dimensional subalgebras $\mathfrak{g} \subset$ $\mathcal{D}^{1}(M)$. In this case, most of the known results on Lie algebra cohomology, [17], are not directly applicable since they apply to cohomology classes having values in finitedimensional modules, whereas in our case the relevant module $\mathcal{Q}$ has finite co-dimension in $\mathcal{F}(M)$.

Based on Theorem 3, there are three basic steps required to classify finite-dimensional Lie algebras of first order differential operators. First, one needs to classify the finite dimensional Lie algebras of vector fields $\mathfrak{h}$ up to changes of variables. Secondly, for each of these Lie algebras, one needs to classify all possible finite-dimensional $\mathfrak{h}$-modules of analytic functions. Trivial modules, valid for any Lie algebra of vector fields, are the zero module $\mathfrak{M}=0$, which consists of the zero function alone, and that containing just the constant functions, $\mathfrak{M}=\mathbb{C}$. (Indeed, for quasi-exactly solvable Lie algebras, these are the only two admissible modules - see below.) Fixing the Lie algebra $\mathfrak{h}$ and the module $\mathfrak{M}$, the first cohomology space $H^{1}(\mathfrak{h}, \mathcal{Q})$ will determine a parametrized system of inequivalent Lie algebras of differential operators. Typically, the cohomology classes are parametrized by one or more continuous parameters or, possibly, analytic functions. In the case of one- 
or two-dimensional complex manifolds, the local classification problem has been solved. The classification of finite-dimensional (non-singular) Lie algebras of vector fields in one and two complex variables appears in Lie, $[\mathbf{2 1}],[\mathbf{2 2}]$. The remaining classification of the finite-dimensional modules $\mathfrak{M}$ and the associated cohomology space $H^{1}(\mathfrak{h}, \mathcal{Q})$ for each of the Lie algebras of vector fields was completed by Miller, [23], and the authors, [19], in one dimension, and by the authors, $[\mathbf{1 0}]$, in two dimensions.

\section{Real Forms of Complex Algebras.}

We now turn to the main topic of this paper, which is the extension of the known classification results for complex Lie algebras of differential operators to the real domain. We begin with a few elementary remarks concerning the complexification of real vector spaces. If $V$ is a real vector space, its complexification is the complex vector space ${ }^{\dagger}$ $V_{\mathbb{C}}=V \otimes \mathbb{C}$. Every element $u \in V_{\mathbb{C}}$ can be written in the form $u=v+i w$, where $v, w \in V$. Thus, $V_{\mathbb{C}}=V \oplus i V$. As usual, we set $v=\operatorname{Re} u, w=\operatorname{Im} u$, and $\bar{u}=v-i w$. If $V$ is finite-dimensional, and $\mathcal{B}=\left\{e_{1}, \ldots, e_{n}\right\}$ is a basis of $V$, then it remains a (real) basis of $V_{\mathbb{C}}$. If $W \subset V$ is any subspace, then its complexification $W_{\mathbb{C}}=W \otimes \mathbb{C}$ is clearly a subspace of $V_{\mathbb{C}}$. We shall say that $W_{\mathbb{C}}$ is a complexified subspace of $V_{\mathbb{C}}$. Not every subspace $\widehat{W} \subset V_{\mathbb{C}}$ is a complexified subspace. For example, if $V=\mathbb{R}^{2}$, so $V_{\mathbb{C}}=\mathbb{C}^{2}$, the one-dimensional subspace $\widehat{W} \subset V_{\mathbb{C}}$ spanned by the vector $(1, i)$ is not a complexified subspace. The following elementary characterization of complexified subspaces of complex vector spaces will play a crucial role in our presentation.

Lemma 4. Let $V_{\mathbb{C}}=V \otimes \mathbb{C}$ be a complexified vector space, and let $W_{\mathbb{C}} \subset V_{\mathbb{C}}$ be a complex subspace. Then the following conditions are equivalent:

(i) $W_{\mathbb{C}}=W \otimes \mathbb{C}$ is a complexified subspace for some $W \subset V$.

(ii) $W_{\mathbb{C}}=\overline{W_{\mathbb{C}}}$,

(iii) $\operatorname{Re} W_{\mathbb{C}}=\operatorname{Im} W_{\mathbb{C}}=W$ are equal, in which case $W_{\mathbb{C}}=W \otimes \mathbb{C}$.

Corollary 5. A finite-dimensional complex subspace $W_{\mathbb{C}} \subset V_{\mathbb{C}}$ of a complexified vector space is a complexified subspace if and only if it admits a real basis $\mathcal{B} \subset V$, which also forms a basis for its real form $W=\operatorname{Re} W_{\mathbb{C}}$. In this case, if $\widehat{\mathcal{B}}=\left\{w_{1}, \ldots, w_{n}\right\}$ forms an arbitrary complex basis for $W_{\mathbb{C}}$, then a real basis of $W_{\mathbb{C}}$ can be found among the elements of $\operatorname{Re} \widehat{\mathcal{B}} \cup \operatorname{Im} \widehat{\mathcal{B}}=\left\{\operatorname{Re} w_{1}, \ldots, \operatorname{Re} w_{n}, \operatorname{Im} w_{1}, \ldots, \operatorname{Im} w_{n} \cdot\right\}$.

Clearly, the basic definitions and results stated in Section 6 can be formulated for real manifolds without any change, other than replacing $\mathbb{C}$ by $\mathbb{R}$ wherever it occurs. Thus, we have the space $\mathcal{F}(M)=\mathcal{F}(M, \mathbb{R})$ of real-valued analytic functions defined on the realanalytic manifold $M$, the space $\mathcal{V}(M)$ of real-analytic vector fields on $M$, and the space $\mathcal{D}^{1}(M)=\mathcal{V}(M) \ltimes \mathcal{F}(M)$ of analytic first-order differential operators $T=\mathbf{v}+f$. In local coordinates $x=\left(x^{1}, \ldots, x^{m}\right)$, the elements of $\mathcal{D}^{1}(M)$ take the usual form

$$
T=\sum_{i=1}^{m} \xi^{i}(x) \frac{\partial}{\partial x^{i}}+f(x),
$$

\footnotetext{
$\dagger$ Tensor products are always over the real field $\mathbb{R}$.
} 
where $\xi^{i}$ and $f$ are real-valued analytic functions on $M$.

In addition to real-valued objects, it will be convenient to consider their complexvalued counterparts, each of which is obtained by complexification. Thus we let $\mathcal{F}(M, \mathbb{C})=$ $\mathcal{F}(M) \otimes \mathbb{C}$ denote the space of complex-valued analytic functions on $M$. A function $f \in$ $\mathcal{F}(M, \mathbb{C})$ can be decomposed into its real and imaginary parts $f(x)=f_{1}(x)+i f_{2}(x)$, where $f_{1}=\operatorname{Re} f, f_{2}=\operatorname{Im} f \in \mathcal{F}(M)$. In quantum mechanics, the wave functions, while defined on a real vector space, are generally complex-valued, and so the appearance here of $\mathcal{F}(M, \mathbb{C})$ will come as no surprise. Similarly, we set $\mathcal{V}(M, \mathbb{C})=\mathcal{V}(M) \otimes \mathbb{C}$ to be the space of complex-valued analytic vector fields on $M$. In local coordinates, an element of $\mathcal{V}(M, \mathbb{C})$ has the form $\mathbf{v}=\sum_{i=1}^{m} \xi^{i}(x) \partial_{x^{i}}$, where the coefficients $\xi^{i} \in \mathcal{F}(M, \mathbb{C})$ are complex-valued analytic functions. Note that $\mathbf{v}=\operatorname{Re} \mathbf{v}+i \operatorname{Im} \mathbf{v}$, where

$$
\operatorname{Re} \mathbf{v}=\sum_{i}\left(\operatorname{Re} \xi^{i}\right) \frac{\partial}{\partial x^{i}}, \quad \operatorname{Im} \mathbf{v}=\sum_{i}\left(\operatorname{Im} \xi^{i}\right) \frac{\partial}{\partial x^{i}}
$$

Finally, $\mathcal{D}^{1}(M, \mathbb{C})=\mathcal{V}(M, \mathbb{C}) \ltimes \mathcal{F}(M, \mathbb{C})=\mathcal{D}^{1}(M) \otimes \mathbb{C}$ is the space of complex-valued analytic first-order differential operators on $M$. Its elements have the same form as in (8), but the coefficient functions $\xi^{i}, f$, are now allowed to be complex-valued.

Assuming analyticity, there is an important connection between the real and complex objects, provided by the inverse procedures of restriction to the real domain and analytic continuation. Since we are dealing with local issues, we can assume, for simplicity, that our differential operators are defined on open subsets of the appropriate real or complex Euclidean space. First, suppose $\widehat{M} \subset \mathbb{C}^{m}$ is an open domain in complex $m$-dimensional Euclidean space, and $\widehat{f}: \widehat{M} \rightarrow \mathbb{C}$ a complex-analytic function. Then the restriction of $\widehat{f}$ to $^{\dagger} M=\widehat{M} \cap \mathbb{R}^{m}$ defines a (in general) complex-valued real-analytic function $f: M \rightarrow \mathbb{C}$. We let $\mathcal{R}: \mathcal{F}(\widehat{M}) \rightarrow \mathcal{F}(M, \mathbb{C})$ denote this restriction map. Conversely, given a complexvalued analytic function $f \in \mathcal{F}(M, \mathbb{C})$ defined on an open subset $M \subset \mathbb{R}^{m}$, its analytic continuation to the complex domain $\mathbb{C}^{m}$ defines a complex-analytic function $\widehat{f}: \widehat{M} \rightarrow \mathbb{C}$ defined on a subdomain $\widehat{M} \subset \mathbb{C}^{m}$ such that $\widehat{M} \cap \mathbb{R}^{m}=M$. (Actually, $\widehat{M} \cap \mathbb{R}^{m}$ may be strictly larger than $M$ if $f$ can be analytically continued as a real-analytic function.) We will always assume that, by suitably restricting the domain $\widehat{M}$, the analytic continuation $\widehat{f}$ is a single-valued function. In our applications, the functions considered are, by and large, combinations of rational and exponential functions, and so the more technical issues associated with the process of analytic continuation do not arise. We let $\mathcal{C}: \mathcal{F}(M, \mathbb{C}) \rightarrow$ $\mathcal{F}(\widehat{M})$ denote the process of analytic continuation, so $\mathcal{C}(f)=\widehat{f}$. Note that the restriction and analytic continuation operators are inverses of each other, meaning that $\mathcal{R} \circ \mathcal{C}=\mathbb{1}$ and $\mathcal{C} \circ \mathcal{R}=\mathbb{1}$, provided $M$ and $\widehat{M}$ are suitably related.

The restriction of a subspace $\widehat{\mathfrak{M}} \subset \mathcal{F}(\widehat{M})$ to the real axis will be a subspace $\mathcal{R}(\widehat{\mathfrak{M}})$ of the space $\mathcal{F}(M, \mathbb{C})$ of complex-valued functions. However, in general $\mathcal{R}(\widehat{\mathfrak{M}})$ does not arise from the complexification of a subspace of real-valued functions, meaning that $\mathcal{R}(\widehat{\mathfrak{M}})$ need not have the form $\mathfrak{M} \otimes \mathbb{C}$ where $\mathfrak{M} \subset \mathcal{F}(M)$ is a real subspace. Lemma 4 shows that this

$\dagger$ We always assume that $\widehat{M} \cap \mathbb{R}^{m} \neq \varnothing$. 
happens if and only if the subspace $\mathcal{R}(\widehat{\mathfrak{M}})$ equals its own complex conjugate. For example, if $\widehat{\mathfrak{M}}$ is generated by the analytic function $z+i$ for $z \in \mathbb{C}$, then its restriction to the real axis is the one-dimensional subspace spanned by the complex function $x+i$, i.e., is equal to the set of all functions of the form $c(x+i)$, where $c$ is an arbitrary complex constant. Here $\overline{\mathcal{R}(\widehat{\mathfrak{M}})}$ is generated by $x-i$, and thus $\mathcal{R}(\widehat{\mathfrak{M}})$ is not a complexified subspace.

Similar considerations provide a correspondence between real-analytic complex-valued vector fields and differential operators defined on $M \subset \mathbb{R}^{m}$ and their complex-analytic counterparts, defined on $\widehat{M} \subset \mathbb{C}^{m}$. The operations of restriction $\mathcal{R}$ and analytic continuation $\mathcal{C}$ are applied component-wise. Thus, for example, the restriction of a complex-analytic differential operator of the form (3) is obtained by restricting its coefficients to depend on real values of $z=x+i y \in \mathbb{C}^{m}$, i.e., we set $y=0$ in the formulae, so that

$$
\mathcal{R}(T)=\mathcal{R}\left[\sum_{i=1}^{m} \xi^{i}(z) \frac{\partial}{\partial z^{i}}+f(z)\right]=\sum_{i=1}^{m} \xi^{i}(x) \frac{\partial}{\partial x^{i}}+f(x), \quad x \in M,
$$

the latter defining a complex-valued real-analytic differential operator on a suitable subdomain $M \subset \mathbb{R}^{m}$, i.e., an element of $\mathcal{D}^{1}(M, \mathbb{C})$. In particular, the restriction of the vector field $\partial_{z}$ to the real axis is merely $\partial_{x}$, not $\frac{1}{2}\left(\partial_{x}+i \partial_{y}\right)$ ! As in the case of functions, the operators $\mathcal{R}$ and $\mathcal{C}$ are inverses of one another when acting on differential operators.

Note that the restriction and analytic continuation operators respect the Lie algebra structure on the space of differential operators; for example, the analytic continuation of the commutator bracket of two differential operators is the bracket of their continuations:

$$
\mathcal{C}[\mathcal{D}, \mathcal{E}]=[\mathcal{C}(\mathcal{D}), \mathcal{C}(\mathcal{E})]
$$

Therefore, if $\widehat{\mathfrak{g}} \subset \mathcal{D}^{1}(\widehat{M})$ is a Lie algebra of complex-analytic first order differential operators on $\widehat{M} \subset \mathbb{C}^{m}$, its restriction to $M \subset \mathbb{R}^{m}$ is the complex Lie algebra $\mathcal{R}(\widehat{\mathfrak{g}})=$ $\{\mathcal{R}(T) \mid T \in \widehat{\mathfrak{g}}\} \subset \mathcal{D}^{1}(M, \mathbb{C})$ defined on the domain $M=\widehat{M} \cap \mathbb{R}^{m}$. The restriction $\mathcal{R}(\widehat{\mathfrak{g}})$ defines a real Lie algebra of differential operators if and only if it is a complexified Lie algebra, so that $\mathcal{R}(\widehat{\mathfrak{g}})=\mathfrak{g}_{\mathbb{C}}=\mathfrak{g} \otimes \mathbb{C}$, where $\mathfrak{g}=\operatorname{Re}\left(\mathfrak{g}_{\mathbb{C}}\right)$ is the associated real Lie algebra. Conversely, given a finite-dimensional ${ }^{\dagger}$ real-analytic Lie algebra of differential operators $\mathfrak{g} \subset \mathcal{D}^{1}(M)$, then there is a corresponding complex-analytic Lie algebra of differential operators $\widehat{\mathfrak{g}}=\mathcal{C}\left(\mathfrak{g}_{\mathbb{C}}\right) \subset \mathcal{D}^{1}(\widehat{M})$, defined on a suitable subdomain $\widehat{M} \subset \mathbb{C}^{m}$, which is obtained by analytically continuing the corresponding complexified Lie algebra $\mathfrak{g}_{\mathbb{C}}=\mathfrak{g} \otimes \mathbb{C}$. Thus we deduce the fundamental correspondence between real and complex Lie algebras of differential operators.

Proposition 6. The analytic continuation of a real-valued finite-dimensional Lie algebra of real-analytic differential operators defines a finite-dimensional Lie algebra of complex-analytic differential operators. Conversely, a Lie algebra of complex-analytic differential operators determines a real Lie algebra of differential operators via restriction if and only if its restriction is a complexified Lie algebra.

$\dagger$ The finite-dimensionality is important here, since otherwise there is no guarantee that the analytic continuation of the individual differential operators have a common domain of definition. 
Two complex Lie algebras of differential operators are equivalent if and only if there is a complex-analytic change of variables and gauge transformation mapping one to the other. Similarly, two real Lie algebras of differential operators are equivalent if and only if there is a real-analytic change of variables and gauge transformation mapping one to the other. Clearly, analytically continuing the complexifications of two equivalent real Lie algebras produces (locally) equivalent complex Lie algebras. The converse, though, is false in general: two real Lie algebras on $M$ whose complexifications have equivalent analytic continuations are not necessarily equivalent.

In general, by a real form of a complex Lie algebra of differential operators $\tilde{\mathfrak{g}}$, we mean any real Lie algebra of differential operators $\mathfrak{g}$ which is obtained by first applying a complex change of variables and gauge transformation, leading to the complex-equivalent algebra $\widehat{\mathfrak{g}}=\Psi(\widetilde{\mathfrak{g}})$, and then restricting to the real axis. Proposition 6 requires that the resulting complex-valued algebra $\mathfrak{g}_{\mathbb{C}}=\mathcal{R}(\widehat{\mathfrak{g}})$ be a complexified algebra, whereby $\mathfrak{g}=\operatorname{Re} \mathfrak{g}_{\mathbb{C}}$, and $\mathfrak{g}_{\mathbb{C}}=\mathfrak{g} \otimes \mathbb{C}$. Two different real forms of a given complex Lie algebra will always be analytically continuable to complex-equivalent Lie algebras, although the real forms may not be real-equivalent (even if they are isomorphic as abstract Lie algebras). For example, the real Lie algebras generated by $\partial_{x}+\partial_{y}, x \partial_{x}+y \partial_{y}, x^{2} \partial_{x}+y^{2} \partial_{y}$, and by $\partial_{x}, x \partial_{x}+y \partial_{y}$, $\left(x^{2}-y^{2}\right) \partial_{x}+2 x y \partial_{y}$ are both isomorphic to $\mathfrak{s l}(2, \mathbb{R})$, and are real forms of the complex Lie algebra with generators $\partial_{z}+\partial_{w}, z \partial_{z}+w \partial_{w}, z^{2} \partial_{z}+w^{2} \partial_{w}$. However, the two real forms are not equivalent - there is no real change of variables mapping one to the other; see [11]. A key problem, then, is to determine the different possible (real-)inequivalent real forms of a given complex Lie algebra of differential operators.

We now describe a general approach to this problem, based on a solution to the complex classification problem. Each complex Lie algebra $\widehat{\mathfrak{g}}$ of differential operators on $\widehat{M} \subset \mathbb{C}^{m}$ can, in accordance with Theorem 3 , be identified with a triple $(\widehat{\mathfrak{h}}, \widehat{\mathfrak{M}},[\widehat{F}])$, where $\widehat{\mathfrak{h}} \subset \mathcal{V}(\widehat{M})$ is a complex Lie algebra of vector fields on $\widehat{M}$. Clearly, if $\widehat{\mathfrak{g}}$ is to restrict to a complexified Lie algebra of differential operators $\mathcal{R}(\widehat{\mathfrak{g}})=\mathfrak{g} \otimes \mathbb{C} \equiv \mathfrak{g}_{\mathbb{C}}$, then $\widehat{\mathfrak{h}}$ must restrict to a complexified Lie algebra of vector fields $\mathcal{R}(\widehat{\mathfrak{h}})=\mathfrak{h} \otimes \mathbb{C} \equiv \mathfrak{h}_{\mathbb{C}} \subset \mathcal{V}(M, \mathbb{C})$. Furthermore, the $\widehat{\mathfrak{h}}$-module $\widehat{\mathfrak{M}} \subset \mathcal{F}(\widehat{M})$ must restrict to a complexified module $\mathcal{R}(\widehat{\mathfrak{M}})=\mathfrak{M}_{\mathbb{C}}=\mathfrak{M} \otimes \mathbb{C} \subset$ $\mathcal{F}(M, \mathbb{C})$. For example, if the complex module $\widehat{\mathfrak{M}}=\mathfrak{M}_{\mathbb{C}}=\mathbb{C}$ consists just of the constants, then $\mathfrak{M}=\mathbb{R}$ also consists of the real constants.

Since the quotient of two complexified spaces is complexified, the quotient space $\mathcal{Q}_{\mathbb{C}}=$ $\mathcal{F}(M, \mathbb{C}) / \mathfrak{M}_{\mathbb{C}}$ is also complexified, with $\mathcal{Q}_{\mathbb{C}}=\mathcal{Q} \otimes \mathbb{C}$, where $\mathcal{Q}=\mathcal{F}(M) / \mathfrak{M}$. Given a complex cocycle $\widehat{F} \in Z^{1}(\widehat{\mathfrak{h}}, \widehat{\mathcal{Q}})$, we define its restriction $F_{\overparen{C}}=\mathcal{R}(\widehat{F})$ to be the complex cocycle defined by

$$
\left\langle F_{\mathbb{C}} ; \mathbf{v}\right\rangle=\mathcal{R}(\langle\widehat{F} ; \mathcal{C}(\mathbf{v})\rangle), \quad \text { for all } \quad \mathbf{v} \in \mathfrak{h}_{\mathbb{C}}
$$

In other words,

$$
F_{\mathbb{C}}=\mathcal{R} \circ \widehat{F} \circ \mathcal{C} \text {. }
$$

The fact that the maps $\mathcal{R}$ and $\mathcal{C}$ are Lie algebra isomorphisms implies that $F_{\mathbb{C}}$ is an element of $Z^{1}\left(\mathfrak{h}_{\mathbb{C}}, \mathcal{Q}_{\mathbb{C}}\right)$. In particular, the complex vector spaces $Z^{1}(\widehat{\mathfrak{h}}, \widehat{\mathcal{Q}})$ and $Z^{1}\left(\mathfrak{h}_{\mathbb{C}}, \mathcal{Q}_{\mathbb{C}}\right)$ are isomorphic:

$$
Z^{1}\left(\mathfrak{h}_{\mathbb{C}}, \mathcal{Q}_{\mathbb{C}}\right)=\mathcal{R} \circ Z^{1}(\widehat{\mathfrak{h}}, \widehat{\mathcal{Q}}) \circ \mathcal{C}
$$


The complex conjugate $\overline{F_{\mathbb{C}}}: \mathfrak{h}_{\mathbb{C}} \rightarrow \overline{\mathcal{Q}_{\mathbb{C}}}=\mathcal{Q}_{\mathbb{C}}$ of a typical element $F_{\mathbb{C}} \in Z^{1}\left(\mathfrak{h}_{\mathbb{C}}, \mathcal{Q}_{\mathbb{C}}\right)$, defined by

$$
\left\langle\overline{F_{\mathbb{C}}} ; \mathbf{v}\right\rangle=\overline{\left\langle F_{\mathbb{C}} ; \overline{\mathbf{v}}\right\rangle}, \quad \text { for all } \quad \mathbf{v} \in \mathfrak{h}_{\mathbb{C}},
$$

is also a cocycle by $(5)$. This shows that the space $Z_{\mathbb{C}}^{1}=Z^{1}\left(\mathfrak{h}_{\mathbb{C}}, \mathcal{Q}_{\mathbb{C}}\right)$ of $\mathcal{Q}_{\mathbb{C}}$-valued cocycles is complexified, so $Z_{\mathbb{C}}^{1}=Z^{1} \otimes \mathbb{C}$, where $Z^{1}=\operatorname{Re}\left(Z_{\mathbb{C}}^{1}\right)$. From (10) it is clear that if $F_{\mathbb{C}} \in Z_{\mathbb{C}}^{1}$ then $\operatorname{Re} F_{\mathbb{C}}$ is a real $\mathcal{Q}$-valued cocycle on $\mathfrak{h}$, and conversely any real cocycle $F \in Z^{1}(\mathfrak{h}, \mathcal{Q})$, when extended by linearity to $\mathfrak{h}_{\mathbb{C}}$, is in $Z_{\mathbb{C}}^{1}$ by $(5)$. Thus, $Z^{1}$ can be identified with $Z^{1}(\mathfrak{h}, \mathcal{Q})$.

Furthermore, if $\delta \sigma$ is the coboundary of a complex-valued function $\sigma \in \hat{\mathcal{Q}}$, then its restriction $(\delta \sigma)_{\mathbb{C}}$ to $Z^{1}\left(\mathfrak{h}_{\mathbb{C}}, \mathcal{Q}_{\mathbb{C}}\right)$, as defined by $(9)$, is the coboundary of $\mathcal{R}(\sigma) \in \mathcal{Q}_{\mathbb{C}}$, since

$$
\begin{aligned}
\left\langle(\delta \sigma)_{\mathbb{C}} ; \mathbf{v}\right\rangle & =\mathcal{R}(\langle\delta \sigma ; \mathcal{C}(\mathbf{v})\rangle)=\mathcal{R}(\mathcal{C}(\mathbf{v})(\sigma)) \quad \text { for all } \quad \mathbf{v} \in \mathfrak{h}_{\mathbb{C}} . \\
& =\mathbf{v}(\mathcal{R}(\sigma))=\langle\delta \mathcal{R}(\sigma) ; \mathbf{v}\rangle,
\end{aligned}
$$

This means that

$$
B^{1}\left(\mathfrak{h}_{\mathbb{C}}, \mathcal{Q}_{\mathbb{C}}\right)=\mathcal{R} \circ B^{1}(\widehat{\mathfrak{h}}, \widehat{\mathcal{Q}}) \circ \mathcal{C}
$$

and the spaces $B^{1}(\widehat{\mathfrak{h}}, \widehat{\mathcal{Q}})$ and $B^{1}\left(\mathfrak{h}_{\mathbb{C}}, \mathcal{Q}_{\mathbb{C}}\right)$ are thus isomorphic. Given an element $\delta \beta \in$ $B^{1}\left(\mathfrak{h}_{\mathbb{C}}, \mathcal{Q}_{\mathbb{C}}\right)$, with $\beta \in \mathcal{Q}_{\mathbb{C}}$, its complex conjugate $\overline{\delta \beta}$ is easily seen to be equal to the coboundary of $\bar{\beta}$. Since $\mathcal{Q}_{\mathbb{C}}$ is a complexified space, this implies that

$$
\overline{\delta \beta}=\delta \bar{\beta} \in \delta\left(\overline{\mathcal{Q}_{\mathbb{C}}}\right)=\delta\left(\mathcal{Q}_{\mathbb{C}}\right)=B^{1}\left(\mathfrak{h}_{\mathbb{C}}, \mathcal{Q}_{\mathbb{C}}\right),
$$

and therefore the space $B_{\mathbb{C}}^{1}=B^{1}\left(\mathfrak{h}_{\mathbb{C}}, \mathcal{Q}_{\mathbb{C}}\right)$ of 1 -coboundaries is also complexified:

$$
B_{\mathbb{C}}^{1}=B^{1} \otimes \mathbb{C},
$$

where as before it is immediate to show that $B^{1}=\operatorname{Re}\left(B_{\overparen{C}}^{1}\right)$ can be identified with $B^{1}(\mathfrak{h}, \mathcal{Q})$. Therefore, the resulting cohomology space $H^{1}\left(\mathfrak{h}_{\mathbb{C}}, \mathcal{Q}_{\mathbb{C}}\right)=Z_{\mathbb{C}}^{1} / B_{\mathbb{C}}^{1} \equiv H_{\mathbb{C}}^{1}$ is isomorphic to $H^{1}(\widehat{\mathfrak{h}}, \widehat{\mathcal{Q}})$ and complexified:

$$
H_{\mathbb{C}}^{1}=\mathcal{R} \circ H^{1}(\widehat{\mathfrak{h}}, \widehat{\mathcal{Q}}) \circ \mathcal{C}=H^{1} \otimes \mathbb{C}, \quad \text { with } \quad H^{1}=H^{1}(\mathfrak{h}, \mathcal{Q}) .
$$

This suffices to prove our basic complexification result:

Theorem 7. Let $\widehat{\mathfrak{h}} \subset \mathcal{V}(\widehat{M})$ be a complex Lie algebra of complex-analytic vector fields, and let $\widehat{\mathfrak{M}} \subset \mathcal{F}(\widehat{M})$ be a complex $\widehat{\mathfrak{h}}$-module of complex-analytic functions. Suppose the restrictions $\mathcal{R}(\widehat{\mathfrak{h}}) \subset \mathcal{V}(M, \mathbb{C})$ and $\mathcal{R}(\widehat{\mathfrak{M}}) \subset \mathcal{F}(M, \mathbb{C})$ are complexified spaces:

$$
\mathcal{R}(\widehat{\mathfrak{h}})=\mathfrak{h} \otimes \mathbb{C} \equiv \mathfrak{h}_{\mathbb{C}}, \quad \mathcal{R}(\widehat{\mathfrak{M}})=\mathfrak{M} \otimes \mathbb{C} \equiv \mathfrak{M}_{\mathbb{C}}
$$

The quotient module $\widehat{\mathcal{Q}}=\mathcal{F}(\widehat{M}) / \widehat{\mathfrak{M}}$ restricts to $\mathcal{R}(\widehat{\mathcal{Q}})=\mathcal{Q}_{\mathbb{C}}=\mathcal{Q} \otimes \mathbb{C}=\mathcal{F}(M, \mathbb{C}) / \mathfrak{M}_{\mathbb{C}}$, with real counterpart $\mathcal{Q}=\mathcal{F}(M) / \mathfrak{M}$. Moreover, the restriction $\mathcal{R} \circ H^{1}(\widehat{\mathfrak{h}}, \widehat{\mathcal{Q}}) \circ \mathcal{C}$ of the associated cohomology space $H^{1}(\widehat{\mathfrak{h}}, \widehat{\mathcal{Q}})$ is also a complexified space, with

$$
\mathcal{R} \circ H^{1}(\widehat{\mathfrak{h}}, \widehat{\mathcal{Q}}) \circ \mathcal{C}=H^{1}\left(\mathfrak{h}_{\mathbb{C}}, \mathcal{Q}_{\mathbb{C}}\right)=H^{1}(\mathfrak{h}, \mathcal{Q}) \otimes \mathbb{C} \equiv H_{\mathbb{C}}^{1} .
$$


In particular, Theorem 7 implies that the real and complex cohomology spaces of a complex Lie algebra of vector fields and any of its real forms have the same dimension:

$$
\operatorname{dim}_{\mathbb{C}} H^{1}(\widehat{\mathfrak{h}}, \widehat{\mathcal{Q}})=\operatorname{dim}_{\mathbb{C}} H^{1}\left(\mathfrak{h}_{\mathbb{C}}, \mathcal{Q}_{\mathbb{C}}\right)=\operatorname{dim}_{\mathbb{R}} H^{1}(\mathfrak{h}, \mathcal{Q}) .
$$

Therefore, the space of complex-inequivalent Lie algebras of differential operators corresponding to a given $\widehat{\mathfrak{h}} \subset \mathcal{V}(\widehat{M})$ and $\widehat{\mathfrak{M}} \subset \mathcal{F}(\widehat{M})$ has the same dimension as the space of real-inequivalent Lie algebras of differential operators corresponding to the real forms $\mathfrak{h} \subset \mathcal{V}(M)$ and $\mathfrak{M} \subset \mathcal{F}(M)$. According to Corollary 5 , if $\mathcal{B}=\left\{\left[F_{1}\right], \ldots,\left[F_{n}\right]\right\}$ forms a basis for the complex cohomology space $H^{1}\left(\mathfrak{h}_{\mathbb{C}}, \mathcal{Q}_{\mathbb{C}}\right)$, then a basis for the real form $H^{1}(\mathfrak{h}, \mathcal{Q})$ can be found among $\operatorname{Re} \mathcal{B} \cup \operatorname{Im} \mathcal{B}=\left\{\left[\operatorname{Re} F_{1}\right], \ldots,\left[\operatorname{Re} F_{n}\right],\left[\operatorname{Im} F_{1}\right], \ldots\left[\operatorname{Im} F_{n}\right]\right\}$. In other words, exactly $n$ of the real and imaginary parts of the complex cocycles $F_{1}, \ldots, F_{n}$ will be linearly independent modulo coboundaries.

Theorem 7 implies that the problem of classifying real Lie algebras of differential operators can be tackled directly as follows. Let $\mathfrak{h}$ be a real form of a complex Lie algebra of vector fields $\widetilde{\mathfrak{h}}$. Let $\varphi: \widetilde{M} \rightarrow \widetilde{M}$ be the change of variables mapping $\mathfrak{h}$ to a complexequivalent Lie algebra $\widehat{\mathfrak{h}}=\varphi(\widetilde{\mathfrak{h}})$, whose restriction coincides with the complexification of our chosen real form: $\mathfrak{h}_{\mathbb{C}}=\mathcal{R}(\widehat{\mathfrak{h}})=\mathfrak{h} \otimes \mathbb{C}$. If $\widetilde{\mathfrak{M}} \subset \mathcal{F}(\widetilde{M})$ is a finite-dimensional $\widetilde{\mathfrak{h}}-$ module of complex-analytic functions, then $\widehat{\mathfrak{M}}=\varphi_{*}(\widetilde{\mathfrak{M}})$ is a finite-dimensional $\widehat{\mathfrak{h}}$-module obtained by applying the change of variables. We assume that its restriction $\mathcal{R}(\widehat{\mathfrak{M}})$ is a complexified module: $\mathfrak{M}_{\mathbb{C}}=\mathcal{R}(\widehat{\mathfrak{M}})=\mathfrak{M} \otimes \mathbb{C}$, with $\mathfrak{M} \subset \mathcal{F}(M)$ a real $\mathfrak{h}$-module. Set $\mathcal{Q}_{\mathbb{C}}=$ $\mathcal{F}(M, \mathbb{C}) / \mathfrak{M}_{\mathbb{C}}=\mathcal{Q} \otimes \mathbb{C}$, with $\mathcal{Q}=\mathcal{F}(M) / \mathfrak{M}$. According to Theorem 7 , the cohomology space $H_{\mathbb{C}}^{1}=H^{1}\left(\mathfrak{h}_{\mathbb{C}}, \mathcal{Q}_{\mathbb{C}}\right)$ is complexified: $H_{\mathbb{C}}^{1}=H^{1} \otimes \mathbb{C}$, where $H^{1}=H^{1}(\mathfrak{h}, \mathcal{Q})$. Any complex Lie algebra of differential operators with vector field part $\widehat{\mathfrak{h}}$, represented by a cohomology class in the space $H^{1}(\widehat{\mathfrak{h}}, \widehat{\mathcal{Q}}) \cong H_{\mathbb{C}}^{1}$, is found by applying $\varphi_{*}$ to a Lie algebra of differential operators represented by an element of $\widetilde{H}^{1}=H^{1}(\widetilde{\mathfrak{h}}, \widetilde{\mathcal{Q}})$, where $\widetilde{\mathcal{Q}}=\mathcal{F}(\widetilde{M}) / \widetilde{\mathfrak{M}}$. Theorem 7 implies that a real basis for $H^{1}$ can be constructed by taking the real and imaginary parts of the elements of a basis for $H^{1}(\widehat{\mathfrak{h}}, \widehat{\mathcal{Q}})$ and restricting ourselves to the real hyperplane. Moreover, (13) implies that the number of real cohomology parameters in $H^{1}$ will be the same as the number of complex cohomology parameters in $H^{1}(\widehat{\mathfrak{h}}, \widehat{\mathcal{Q}}) \cong H_{\mathbb{C}}^{1}$. In summary, we can produce a basis for the real cohomology space $H^{1}(\mathfrak{h}, \mathcal{Q})$ by the following procedure: $i$ ) apply the change of variables $\varphi$ to a basis for the original cohomology space $\left.H^{1}(\widetilde{\mathfrak{h}}, \widetilde{\mathcal{Q}}), i i\right)$ restrict the resulting cohomology classes to the real subspace, and $\left.i i i\right)$ choose half of the real and imaginary components of these restricted cohomology classes to form a basis. Note that all the steps in this procedure are totally algorithmic, although in the third step one should bear in mind that we are dealing with equality up to a coboundary. Typically, for each cocycle representing a nontrivial cohomology class in the complex basis, either its real part, or its imaginary part, provides one element of the corresponding real basis. This procedure will be illustrated by the examples in Section 5 .

\section{Quasi-Exact Solvability.}

A finite-dimensional Lie algebra $\mathfrak{g} \subset \mathcal{D}^{1}(M)$ is called quasi-exactly solvable if it admits a non-zero finite-dimensional module (or representation space) $\mathfrak{N} \subset \mathcal{F}(M)$ consisting of 
functions on $M$. In other words, we have $T(h) \in \mathfrak{N}$ whenever $T \in \mathfrak{g}$ and $h \in \mathfrak{N}$. The condition of quasi-exact solvability has one elementary consequence that simplifies our classification procedure. We state this result for complex Lie algebras, although the real version is also immediate.

Proposition 8. If $\mathfrak{g}$ is a quasi-exactly solvable Lie algebra represented by the triple $(\mathfrak{h}, \mathfrak{M},[F])$ as in Theorem 3 , then the module $\mathfrak{M}$ of multiplication operators is either trivial, $\mathfrak{M}=0$, or consists of constants, $\mathfrak{M}=\mathbb{C}$. Moreover, if $\mathfrak{g}=(\mathfrak{h}, 0,[F])$, then $\mathfrak{g} \subset \widetilde{\mathfrak{g}}=\mathfrak{g} \oplus \mathbb{C}$, where $\widetilde{\mathfrak{g}}=(\mathfrak{h}, \mathbb{C},[F])$ is a quasi-exactly solvable central extension of $\mathfrak{g}$.

Therefore, we can assume, without loss of generality, that the only multiplication operators in our Lie algebra are the constant functions, i.e., $\mathfrak{g} \cap \mathcal{F}(M)=\mathbb{C}$. The quotient space $\mathcal{Q}=\mathcal{F}(M) / \mathbb{C}$ is also fixed in this case.

In $[\mathbf{8}],[\mathbf{1 4}]$, a complete classification of all quasi-exactly solvable Lie algebras of firstorder differential operators on $\mathbb{C}^{2}$ was found. A remarkable consequence of the general classification is that the quasi-exact solvability condition imposes an additional "quantization" condition on the cohomology parameters associated with the cohomology space $H^{1}(\mathfrak{h}, \mathcal{Q})$, meaning that these parameters must take on a discrete (e(i)ntegral) set of values. (In [7] this result was seen, in certain cases, to be a consequence of some fundamental algebro-geometric results governing the classification of vector bundles over algebraic varieties.) In the present work, we shall extend the classification to real Lie algebras of differential operators. The basic remark is that the quasi-exact solvability condition is respected by the process of complexification.

Proposition 9. Let $\widehat{M} \subset \mathbb{C}^{m}$ be an open subset, and let $\widehat{\mathfrak{g}} \subset \mathcal{D}^{1}(\widehat{M})$ be a Lie algebra of complex-analytic first-order differential operators. Let $M=\widehat{M} \cap \mathbb{R}^{m}$, and assume that the restriction $\mathcal{R}(\widehat{\mathfrak{g}})$ is a complexified Lie algebra of differential operators, so that $\mathcal{R}(\widehat{\mathfrak{g}})=\mathfrak{g} \otimes \mathbb{C} \equiv \mathfrak{g}_{\mathbb{C}}$ for some real Lie algebra $\mathfrak{g} \subset \mathcal{D}^{1}(M)$. Then all three Lie algebras $\widehat{\mathfrak{g}}, \mathfrak{g}_{\mathbb{C}}, \mathfrak{g}$ are quasi-exactly solvable if and only if any one of them is quasi-exactly solvable.

Proof: First, if $\mathfrak{N} \subset \mathcal{F}(M)$ is a finite-dimensional module for $\mathfrak{g}$, then its complexification $\mathfrak{N}_{\mathbb{C}}=\mathfrak{N} \otimes \mathbb{C}$ is a finite-dimensional module for $\mathfrak{g}_{\mathbb{C}}$, and thus its analytic continuation $\widehat{\mathfrak{N}}=\mathcal{C}\left(\mathfrak{N}_{\mathbb{C}}\right)$ is a finite-dimensional module for $\widehat{\mathfrak{g}}$. Conversely, if $\widehat{\mathfrak{N}}$ is a finite-dimensional module for $\widehat{\mathfrak{g}}$, then its restriction $\mathcal{R}(\widehat{\mathfrak{N}})$ to $M$ is a finite-dimensional module for $\mathfrak{g}_{\mathbb{C}}$. If $\mathcal{R}(\widehat{\mathfrak{N}})=\mathfrak{N} \otimes \mathbb{C}$ is a finite-dimensional complexified $\mathfrak{g}_{\mathbb{C}}$-module, then it immediately follows that $\mathfrak{N}$ is a finite-dimensional $\mathfrak{g}$-module. Otherwise, we just replace $\mathcal{R}(\widehat{\mathfrak{N}})$ by $\mathcal{R}(\widehat{\mathfrak{N}})+\overline{\mathcal{R}}(\widehat{\mathfrak{N}})$, which is also a finite-dimensional $\mathfrak{g}_{\mathbb{C}}$-module and is obviously complexified. Q.E.D.

Thus, the classification of real quasi-exactly solvable Lie algebras can be based on the complex classification. The only question is whether the quantization conditions required for quasi-exact solvability produce, upon restriction, complexified lie algebras or not. This will be the case if and only if the quasi-exact solvability imposes real constraints on the real cohomology parameters. For example, in the planar cases appearing in the complex classification Tables 1, 2 and 3, the quantization conditions are all real, and hence the complex Lie algebra and its restriction obey the same quasi-exact solvability criteria. However, as we shall see, this is not the case for the other real forms of our 
complex Lie algebras, so that the real quasi-exact solvability criteria turn out to be more restrictive than their complex counterparts. Thus, in these cases, to determine the real quasi-exactly solvable Lie algebras of differential operators, we need only determine how the change of variables has affected the quantization condition. If the quantized values for the cohomology parameters remain real under the change of variables, then the real Lie algebra of differential operators will admit a finite-dimensional (complex) module. If, on the other hand, the quantized cohomology condition is no longer real in the new variables, then the real Lie algebra of vector fields will have no real quasi-exactly solvable Lie algebras with nontrivial cohomology.

\section{The Planar Case.}

Let us review what is known in the particular case of two dimensions. In the complex case, there are, up to local changes of variables, a wide variety of nonsingular Lie algebras of vector fields, first classified by Lie, $[\mathbf{2 1}]$. These correspond to the locally inequivalent finite-dimensional transformation group actions on a two-dimensional manifold; the nonsingularity condition avoids points where all of the vector fields simultaneously vanish, i.e., the transformation group has no zero-dimensional orbits. The classification naturally distinguishes between the imprimitive Lie algebras, for which there exists an invariant foliation of the manifold, and the primitive algebras, having no such foliation. Since the construction of quasi-exactly solvable Schrödinger operators requires the associated group action to be transitive, we shall ignore the intransitive cases here. In [10], Lie's classification was reorganized into 24 different categories, although these in turn can be further simplified into 14 categories as given in the tables at the end of the paper, which are adapted from those appearing in [25], and form a simplified version of the classification appearing in our earlier papers $[\mathbf{8}],[\mathbf{1 0}],[14]$. In their canonical forms, each of these Lie algebras appearing in the complex classification is a complexified algebra, and hence has an obvious real counterpart, obtained by restricting the coordinates to be real. Moreover, according to Theorems 7 and 9 , in such cases the associated real Lie algebras of differential operators and finite-dimensional modules are readily obtained by restriction. Interestingly, every imprimitive real Lie algebra is obtained by this simple procedure. In addition to these real Lie algebras, there are precisely five additional primitive real Lie algebras of vector fields in two dimensions that are are not equivalent to any of the Lie algebras obtained by straightforward restriction of the complex normal forms. However, the complexification (or analytic continuation) of each of these five additional Lie algebras will, of course, be equivalent, under a complex diffeomorphism, to one of the complex normal forms on our list. The complete list of these additional real forms along with their canonical complexification appear in Table 4; the required changes of variables needed to change them into the canonical form appears later in this section. Therefore, to complete the classification of all real Lie algebras of first-order differential operators, we need only determine the real cohomology spaces associated with these five additional real forms, and, further, to determine what values of the cohomology parameters will produce quasi-exactly solvable algebras. Combining these results with our earlier complex classification will thus produce a complete list of normal forms for the Lie algebras of first order differential operators in two real variables admitting a finite-dimensional module of smooth functions. 
For simplicity, in the ensuing discussion, the complex $\mathfrak{h}$-module will be always taken to be $\mathfrak{M}_{C}=\mathbb{C}$, as it will in any quasi-exactly solvable algebra, by Proposition 8 . (The general $\mathfrak{g}$-modules and associated cohomologies appear in Tables 5 and 6.) We will use $z, w$ for the complex canonical coordinates, and $x, y$ for the associated real coordinates.

Case 4.1: The first of the real forms is the unimodular algebra with generators

$$
\partial_{x}, \quad x \partial_{x}+y \partial_{y}, \quad\left(x^{2}-y^{2}\right) \partial_{x}+2 x y \partial_{y} .
$$

The complex normal form of this Lie algebra is of Type 1.1, with generators

$$
\partial_{z}+\partial_{w}, \quad z \partial_{z}+w \partial_{w}, \quad z^{2} \partial_{z}+w^{2} \partial_{w} .
$$

Indeed, the change of variables

$$
z=x+i y, \quad w=x-i y,
$$

maps the generators (15) directly to (14). The cohomology space of (15) is one-dimensional, and is represented by the Lie algebra of differential operators spanned by

$$
\partial_{z}+\partial_{w}, \quad z \partial_{z}+w \partial_{w}, \quad z^{2} \partial_{z}+w^{2} \partial_{w}+c(z-w) .
$$

Applying the change of variables (16) to the associated differential operators, we find that the real cohomology is also one-dimensional (in accordance with Lemma 4) and represented by

$$
\partial_{x}, \quad x \partial_{x}+y \partial_{y}, \quad\left(x^{2}-y^{2}\right) \partial_{x}+2 x y \partial_{y}+b y,
$$

where the real cohomology parameter is related to the complex one via $b=2 i c$. Finally, the complex quantization condition requires that $c=\frac{1}{2} n, n \in \mathbb{N}$, is a half integer. For the real Lie algebra (18), this requires that $b=i n$ be complex, which makes the Lie algebra no longer complexified. We conclude that there are no real quasi-exactly solvable Lie algebras with nonzero cohomology.

Thus, the only Lie algebra of this form having a nontrivial module is the original vector field model (14). In this case, the fundamental complex $\mathfrak{g}$-modules are of the form

$$
\mathfrak{N}_{m}=\operatorname{Span}\left\{\psi_{k}^{m} \mid 0 \leq k \leq 2 m\right\},
$$

with $m \in \mathbb{N}$ a nonnegative integer,

$$
\psi_{k}^{m}(x, y)=y^{m-k} R_{k}^{m}\left(\frac{x}{i y}\right), \quad R_{k}^{m}(t)=\frac{d^{k}}{d t^{k}}\left(t^{2}-1\right)^{m} .
$$

It is easy to see that $\mathfrak{N}_{m}$ is a complexified module for all $m \in \mathbb{N}$. Indeed, a straightforward calculation shows that the functions

$$
i^{k} \psi_{k}^{m}=k !(-1)^{m-k} \sum_{\frac{k}{2} \leq j \leq m}\left(\begin{array}{c}
m \\
j
\end{array}\right)\left(\begin{array}{c}
2 j \\
k
\end{array}\right) x^{2 j-k} y^{m-2 j}, \quad k=0, \ldots, 2 m,
$$


form a real basis thereof. Every other finite-dimensional real module is a direct sum of the fundamental real modules $\operatorname{Re} \mathfrak{N}_{m}$.

Case 4.2: The second case to consider is the orthogonal Lie algebra generated by

$$
\mathbf{v}_{1}=\left(1+x^{2}-y^{2}\right) \partial_{x}+2 x y \partial_{y}, \quad \mathbf{v}_{2}=-y \partial_{x}+x \partial_{y}, \quad \mathbf{v}_{3}=2 x y \partial_{x}+\left(1-x^{2}+y^{2}\right) \partial_{y} .
$$

The group action is obtained by stereographic projection of the standard rotation group action on the two-sphere. The complex normal form is again of Type 1.1, as in (15), with one-dimensional cohomology, as in (17). Under the change of variables

$$
z=x+i y, \quad w=\frac{-1}{x-i y}=-\frac{x+i y}{x^{2}+y^{2}},
$$

we find

$$
\partial_{z}+\partial_{w}=\frac{1}{2}\left(\mathbf{v}_{1}-i \mathbf{v}_{3}\right), \quad z \partial_{z}+w \partial_{w}=-i \mathbf{v}_{2}, \quad z^{2} \partial_{z}+w^{2} \partial_{w}=\frac{1}{2}\left(\mathbf{v}_{1}+i \mathbf{v}_{3}\right) .
$$

Applying the change of variables (22) to the associated differential operators (17), we find that the complex cohomology is represented by a one-parameter family of cocycles, which we denote by $F_{c}, c \in \mathbb{C}$, with representative Lie algebras

$$
\begin{aligned}
& \left(1+x^{2}-y^{2}\right) \partial_{x}+2 x y \partial_{y}+c(x+i y) \frac{x^{2}+y^{2}+1}{x^{2}+y^{2}}, \\
& 2 x y \partial_{x}+\left(1-x^{2}+y^{2}\right) \partial_{y}-i c(x+i y) \frac{x^{2}+y^{2}+1}{x^{2}+y^{2}},
\end{aligned}
$$

Note that, in contrast to the first case, $F_{c}$ is not a real cocycle for any specialization of the parameter $c$. However, its real and imaginary parts, $\operatorname{Re} F_{c}$ and $\operatorname{Im} F_{c}$ for $c \in \mathbb{R}$, are themselves cocycles. Moreover, according to Theorem 7, only one of these two can form an independent cohomology class in $Z^{1}(\mathfrak{h}, \mathcal{Q})$. Indeed, $\operatorname{Re} F_{c}$ is cohomologous to the zero cocycle since it equals the coboundary of the function $\frac{1}{2} c \log \left(x^{2}+y^{2}\right)$. The other cocycle $\operatorname{Im} F_{c}$ therefore represents the complete real cohomology space which, like its complex counterpart, must be one-dimensional. The latter cocycle can be further simplified by adding in the coboundary of $-c \arctan (y / x)$, leading to the real cohomology representative

$$
\left(1+x^{2}-y^{2}\right) \partial_{x}+2 x y \partial_{y}+b y, \quad-y \partial_{x}+x \partial_{y}, \quad 2 x y \partial_{x}+\left(1-x^{2}+y^{2}\right) \partial_{y}-b x,
$$

where the real cohomology parameter is related to the complex one via $b=2 i c$. In the original complex coordinates, this cocycle can also be obtained by first adding in the coboundary corresponding to $c \log (-w)$, which effectively replaces $\partial_{w}$ by $\partial_{w}+c / w$, leading to the alternative complex cohomology representative

$$
\partial_{z}+\partial_{w}+\frac{c}{w}, \quad z \partial_{z}+w \partial_{w}, \quad z^{2} \partial_{z}+w^{2} \partial_{w}+c z .
$$

As in Case 4.1, the complex quantization condition is $c=\frac{1}{2} n, n \in \mathbb{N}$, which requires that $b=i n$. Consequently, there are no real quasi-exactly solvable Lie algebras with nonzero cohomology. 
As before, the only Lie algebra having a nontrivial module is the original vector field model (21). The fundamental $\mathfrak{s o}(3)$-modules are given by

$$
\mathfrak{N}_{m}=\operatorname{Span}\left\{\phi_{k}^{m} \mid 0 \leq k \leq 2 m\right\}, \quad m \in \mathbb{N},
$$

where

$$
\phi_{k}^{m}(x, y)=\left(\frac{1+x^{2}+y^{2}}{x-i y}\right)^{m-k} R_{k}^{m}\left(\frac{x^{2}+y^{2}-1}{x^{2}+y^{2}+1}\right) .
$$

Let us show, first of all, that all the modules (25) are complexified modules. Indeed, from the identity

$$
R_{k}^{m}(t)=\frac{k !}{(2 m-k) !}\left(t^{2}-1\right)^{m-k} R_{2 m-k}^{m},
$$

cf. $[9 ;(3.15)]$, it follows that

$$
\overline{\phi_{k}^{m}}=(-1)^{m-k} 2^{2(m-k)} \frac{k !}{(2 m-k) !} \phi_{2 m-k}^{m} .
$$

In particular, $\phi_{m}^{m}$ is real. Therefore $\mathfrak{N}_{m}$ admits the real basis

$$
\left\{\operatorname{Re} \phi_{0}^{m}, \ldots, \operatorname{Re} \phi_{m-1}^{m}, \operatorname{Im} \phi_{0}^{m}, \ldots, \operatorname{Im} \phi_{m-1}^{m}, \phi_{m}^{m}\right\}
$$

and is therefore a complexified module of dimension $2 m+1$, whose real counterpart is spanned by the same basis functions. The modules (25) are all irreducible; indeed, it is not hard to see that $\mathfrak{N}_{m}$ is the carrier of the standard $\mathfrak{s o}(3)$ representation with integer spin $m$. See $[9 ;$ pp. $62-63]$ for the precise connection between the functions $(26)$ and the classical spherical harmonics.

It is also of interest to compare our results with those of Morozov et al., [24]. First, we note that the change of variables

$$
\xi=-\frac{2 x}{x^{2}+y^{2}-1}, \quad \eta=\frac{2 y}{x^{2}+y^{2}-1},
$$

defines a diffeomorphism from the complement of the closed unit disc in the $(x, y)$ plane onto the entire $(\xi, \eta)$ plane, and maps the generators (21) into constant multiples of the differential operators

$$
\eta \frac{\partial}{\partial \xi}-\xi \frac{\partial}{\partial \eta}, \quad \xi \eta \frac{\partial}{\partial \xi}+\left(1+\eta^{2}\right) \frac{\partial}{\partial \eta}, \quad\left(1+\xi^{2}\right) \frac{\partial}{\partial \xi}+\xi \eta \frac{\partial}{\partial \eta},
$$

which is precisely the basis of $\mathfrak{s o}(3)$ used in $[24]$. In the new $(\xi, \eta)$ coordinates, the basis elements $(26)$ of the fundamental $\mathfrak{s o}(3)$-module $\mathfrak{N}_{m}$ are expressed as follows:

$$
\phi_{k}^{m}(\xi, \eta)=(-2)^{m-k}(\xi+i \eta)^{k-m} \zeta^{k-m} R_{k}^{m}(\zeta)
$$

where

$$
\zeta=\left(1+\xi^{2}+\eta^{2}\right)^{-1 / 2}=\frac{x^{2}+y^{2}-1}{x^{2}+y^{2}+1}
$$


The $\mathfrak{s o}(3)$-modules considered in $[24]$ are $\tilde{\mathfrak{N}}_{m}=\zeta^{m} \mathcal{P}^{(m)}$, where $\mathcal{P}^{(m)}$ denotes the set of polynomials $P(\xi, \eta)$ of degree $\leq m$. However, as noted in [24], these modules are certainly not irreducible. Indeed, it can be shown that

$$
\tilde{\mathfrak{N}}_{m}=\bigoplus_{i=0}^{\left[\frac{m}{2}\right]} \mathfrak{N}_{m-2 i}
$$

where $\mathfrak{N}_{m}$ is the module spanned by $(31)$, forms the decomposition of $\tilde{\mathfrak{N}}_{m}$ into irreducible summands.

Case 4.3: Consider next the one-parameter family of three-dimensional solvable Lie algebras generated by the vector fields

$$
\partial_{x}, \quad \partial_{y}, \quad \beta\left(x \partial_{x}+y \partial_{y}\right)+y \partial_{x}-x \partial_{y}
$$

where $\beta$ is a real constant. The complex normal form has generators

$$
\partial_{z}, \quad \partial_{w}, \quad(\beta-i) z \partial_{z}+(\beta+i) w \partial_{w},
$$

spanning a Lie algebra of Type 1.7 with $r=1$ and constant $\alpha=(\beta+i) /(\beta-i)$. Indeed, under the change of variables (16), we have

$$
\begin{gathered}
\partial_{z}=\frac{1}{2}\left[\partial_{x}+i \partial_{y}\right], \quad \partial_{w}=\frac{1}{2}\left[\partial_{x}-i \partial_{y}\right] \\
(\beta-i) z \partial_{z}+(\beta+i) w \partial_{w}=\beta\left(x \partial_{x}+y \partial_{y}\right)+y \partial_{x}-x \partial_{y}
\end{gathered}
$$

If $\alpha \notin \mathbb{Q}^{-}$, then our Lie algebra of Type 1.7 has zero cohomology; thus, for real $\beta$, the only case when (32) has nonzero cohomology is when $\beta=0$, corresponding to $\alpha=-1$. In this case, since we are assuming that the module is $\mathfrak{M}=\mathbb{C}$, the cohomology space is one-dimensional, and is represented by the Lie algebra of differential operators spanned by

$$
\partial_{z}, \quad \partial_{w}+c z, \quad z \partial_{z}-w \partial_{w} .
$$

Applying the change of variables (16), we find that the complex cohomology is represented by the family of Lie algebras

$$
\partial_{x}+c x+i c y, \quad \partial_{y}+c y-i c x, \quad-y \partial_{x}+x \partial_{y},
$$

for $c \in \mathbb{C}$. As with Case 4.2, the real part of this cocycle forms a coboundary, while the imaginary part determines the real cohomology basis representative:

$$
\partial_{x}+b y, \quad \partial_{y}-b x, \quad-y \partial_{x}+x \partial_{y} .
$$

The real cohomology parameter is related to the complex one via $b=i c$. There are no complex quasi-exactly solvable algebras with nonzero cohomology in this case, and hence no real ones either. 
For the complex Lie algebra (33), the most general module is a sum of the basic modules

$$
\widehat{\mathfrak{N}}_{r, s}=\operatorname{Span}\left\{z^{j} w^{k} \mid 0 \leq j \leq r, 0 \leq k \leq s\right\}, \quad r, s \in \mathbb{N} .
$$

Applying the change of variables (16), we see that the basic complex modules for the Lie algebra (32) are of the form

$$
\mathfrak{N}_{r, s}=\operatorname{Span}\left\{(x+i y)^{j}(x-i y)^{k} \mid 0 \leq j \leq r, 0 \leq k \leq s\right\}, \quad r, s \in \mathbb{N} .
$$

Since $\overline{\mathfrak{N}_{r, s}}=\mathfrak{N}_{s, r}$, the most general complexified $\mathfrak{g}$-module in this case is a sum of the indecomposable modules $\mathfrak{N}_{r, s}+\mathfrak{N}_{s, r}$. Let $\mathcal{H}^{(m)}$ denote the space of harmonic polynomials of degree at most $m$. The corresponding real indecomposable modules are therefore

$$
\begin{aligned}
\mathfrak{N}_{r, s}^{\mathbb{R}} & =\operatorname{Re}\left(\mathfrak{N}_{r, s}+\mathfrak{N}_{s, r}\right) \\
& =\operatorname{Span}\left\{\left(x^{2}+y^{2}\right)^{j} P_{k}(x, y) \mid P_{k} \in \mathcal{H}^{(k)}, 0 \leq j \leq r, 0 \leq k \leq s-j\right\}, \quad 0 \leq r \leq s .
\end{aligned}
$$

Case 4.4: The four-dimensional real Lie algebra generated by

$$
\partial_{x}, \quad \partial_{y}, \quad x \partial_{x}+y \partial_{y}, \quad y \partial_{x}-x \partial_{y},
$$

has complex normal form of Type 1.9 for $r=1$, with generators

$$
\partial_{z}, \quad \partial_{w}, \quad z \partial_{z}, \quad w \partial_{w} .
$$

This case is completely trivial, since the complex cohomology space is zero-dimensional, and hence there is no real cohomology either. The fundamental complex modules are again given by (38), with their real counterparts being (39).

Case 4.5: Finally, the six-dimensional pseudo-orthogonal Lie algebra $\mathfrak{s o}(3,1)$ is realized on $\mathbb{R}^{2}$ by the generators

$$
\begin{gathered}
\mathbf{v}_{1}=\partial_{x}, \quad \mathbf{v}_{2}=\partial_{y}, \quad \mathbf{v}_{3}=x \partial_{x}+y \partial_{y}, \quad \mathbf{v}_{4}=-y \partial_{x}+x \partial_{y}, \\
\mathbf{v}_{5}=\left(x^{2}-y^{2}\right) \partial_{x}+2 x y \partial_{y}, \quad \mathbf{v}_{6}=2 x y \partial_{x}+\left(y^{2}-x^{2}\right) \partial_{y} .
\end{gathered}
$$

The complex normal form is of Type 1.4, with generators

$$
\partial_{z}, \quad \partial_{w}, \quad z \partial_{z}, \quad w \partial_{w}, \quad z^{2} \partial_{z}, \quad w^{2} \partial_{w},
$$

spanning the Lie algebra $\mathfrak{s o}(4, \mathbb{C}) \cong \mathfrak{s l}(2) \oplus \mathfrak{s l}(2)$. The complex cohomology space for $(43)$ is two-dimensional, and is represented by

$$
\partial_{z}, \quad \partial_{w}, \quad z \partial_{z}, \quad w \partial_{w}, \quad z^{2} \partial_{z}+c_{1} z, \quad w^{2} \partial_{w}+c_{2} w .
$$

Under the change of variables (16), we note that

$$
z^{2} \partial_{z}=\mathbf{v}_{5}+i \mathbf{v}_{6}, \quad w^{2} \partial_{w}=\mathbf{v}_{5}-i \mathbf{v}_{6} .
$$


Therefore, the real cohomology is also two-dimensional, and represented by

$$
\begin{array}{cl}
\partial_{x}, \quad \partial_{y}, \quad x \partial_{x}+y \partial_{y}, & -y \partial_{x}+x \partial_{y}, \\
\left(x^{2}-y^{2}\right) \partial_{x}+2 x y \partial_{y}+b_{1} x+b_{2} y, & 2 x y \partial_{x}+\left(y^{2}-x^{2}\right) \partial_{y}-b_{2} x+b_{1} y
\end{array}
$$

The real cohomology parameters are related to the complex ones via

$$
b_{1}=c_{1}+c_{2}, \quad b_{2}=i\left(c_{1}-c_{2}\right) .
$$

Finally, the complex quantization conditions $c_{1}=-n_{1}, c_{2}=-n_{2}, n_{1}, n_{2} \in \mathbb{N}$, require that the real parameters assume the values $b_{1}=-2 n, b_{2}=0$, where $n=n_{1}=n_{2}$. Therefore, this real Lie algebra of vector fields determines a nontrivial family of quasi-exactly solvable Lie algebras of differential operators, parametrized by the integral cohomology parameter $n$. This finishes the proof of the following theorem.

Theorem 10. Among the five additional real Lie algebras of planar vector fields in $\mathbb{R}^{2}$, the only one admitting a nonzero real-valued quasi-exactly solvable cohomology class is (a central extension of ) $\mathfrak{s o}(3,1)$, for which the corresponding Lie algebra of first-order differential operators is spanned by

$$
\begin{gathered}
T^{0}=1, \quad T^{1}=\partial_{x}, \quad T^{2}=\partial_{y}, \quad T^{3}=x \partial_{x}+y \partial_{y}, \quad T^{4}=y \partial_{x}-x \partial_{y}, \\
T^{5}=\left(x^{2}-y^{2}\right) \partial_{x}+2 x y \partial_{y}-2 n x, \quad T^{6}=2 x y \partial_{x}+\left(y^{2}-x^{2}\right) \partial_{y}-2 n y,
\end{gathered}
$$

where $n$ is a non-negative integer.

In this case, the associated complex module is the polynomial module $\mathfrak{N}_{n, n}$, as defined by (38); see also (39) for its real counterpart. Finally, we note the remarkable fact that all the preceding cases form subalgebras of the latter case.

Theorem 11. Every finite-dimensional Lie algebra of first order differential operators with vector field part given by one of the five additional primitive real forms listed in Table 4 is a subalgebra of one of the pseudo-orthogonal Lie algebras given by (45).

\section{New Quasi-Exactly Solvable $\mathfrak{s o}(3,1)$ Potentials.}

Now that we have completed the classification of real quasi-exactly solvable Lie algebras of differential operators, we can apply the additional canonical forms to construct new quasi-exactly solvable quantum Hamiltonians in two variables. We begin by recalling the basic connection between Lie algebras of first-order differential operators and quasi-exactly solvable quantum systems. Let $M$ be a real or complex manifold. The algebra $\mathcal{D}^{*}(M)$ consisting of all differential operators on $M$ can be identified with the universal enveloping algebra of the space $\mathcal{D}^{1}(M)$ of first-order differential operators. There is a natural filtration of $\mathcal{D}^{*}(M)$ by the subspaces $\mathcal{D}^{n}(M)$ consisting of all differential operators of order at most $n$; equivalently, we may define $\mathcal{D}^{n}(M)$ as the space of all polynomials of degree at most $n$ in the first-order differential operators. In local coordinates, a typical element of $\mathcal{D}^{n}(M)$ can be written in the form

$$
L=\sum_{0 \leq j_{1}+\cdots+j_{m} \leq n} b_{j_{1} \ldots j_{m}}(z) \frac{\partial^{j_{1}+\cdots+j_{m}}}{\left(\partial z^{1}\right)^{j_{1}} \ldots\left(\partial z^{m}\right)^{j_{m}}} .
$$

In physical applications, the differential operator is required to be Hermitian and real. 
Definition 12. A differential operator is called Lie-algebraic if it lies in the universal enveloping algebra of a finite-dimensional subalgebra $\mathfrak{g} \subset \mathcal{D}^{1}(M)$

In other words, any Lie-algebraic operator $L$ can be written as a polynomial (with constant coefficients) in the generators $T^{a}$, which are first order differential operators, of the Lie algebra $\mathfrak{g}$.

Definition 13. A differential operator is called quasi-exactly solvable if it is Liealgebraic for a quasi-exactly solvable Lie algebra $\mathfrak{g} \subset \mathcal{D}^{1}(M)$.

In this case, the associated finite-dimensional module $\mathfrak{N} \subset \mathcal{F}(M)$ is invariant under the quasi-exactly solvable operator $L$, so that $L(\mathfrak{N}) \subset \mathfrak{N}$, and hence $L$ restricts to define a finite-dimensional matrix operator on $\mathfrak{N}$, whose eigenvalues will (provide the functions in $\mathfrak{N}$ are normalizable) form the algebraic part of the spectrum of $L$.

We are interested in constructing examples of quasi-exactly solvable Schrödinger operators. By a Schrödinger operator we mean a second order differential operator of the form

$$
H=-\Delta+V(x)
$$

here

$$
\Delta=\sum_{i, j=1}^{m} g^{i j}\left(x^{1}, \ldots, x^{m}\right) \nabla_{i} \nabla_{j},
$$

is the Laplacian (kinetic energy) operator on the finite-dimensional real Riemannian manifold $M$ with contravariant metric $\left(g^{i j}\right)$, and $\nabla_{i}$ is the covariant derivative associated to this metric. (The physical units are taken so that $\hbar=2 m=1$.) Let $\mathfrak{g}$ be one of our real quasi-exactly solvable Lie algebras of first order differential operators written in canonical form. The most general (real) second-order differential operator $L$ which is quasi-exactly solvable with hidden symmetry algebra $\mathfrak{g}$ takes the form

$$
L=\sum_{a, b=1}^{r} C_{a b} T^{a} T^{b}+\sum_{a=1}^{r} C_{a} T^{a}+C_{0},
$$

where the $T^{a}$ are the generators of $\mathfrak{g}$, and $C_{a b}, C_{a}$ are real constants. Now, in general $L$ need not be a Schrödinger operator (47); however, $L$ can sometimes be transformed into a Schrödinger operator by applying a suitable change of variables $\varphi$ and gauge transformation $\mathcal{G}_{\sigma}$. The transformed operator $L=e^{-\sigma} \circ \varphi_{*}(L) \circ e^{\sigma}$ is then a quasi-exactly solvable operator with respect to the transformed algebra $\mathcal{G}_{\sigma} \circ \varphi_{*}(\mathfrak{g}) \cong \mathfrak{g}$, with generators $\widetilde{T}^{a}=\mathcal{G}_{\sigma} \circ \varphi_{*}\left(T^{a}\right)$, and so, under the extra assumption that the transformed wave functions are normalizable, a finite sector of its spectrum can be algebraically computed.

We recall now the necessary and sufficient conditions under which the operator (48) can be transformed into a Schrödinger operator $H$, cf. for example [13]. First of all, we rewrite $L$ in the form

$$
L=-\sum_{i, j=1}^{m} g^{i j}(x) \frac{\partial^{2}}{\partial x^{i} \partial x^{j}}+\sum_{i=1}^{m} b^{i}(x) \frac{\partial}{\partial x^{i}}+c(x) .
$$


We must first require that $L$ be elliptic, meaning that the quadratic form associated to the symmetric matrix $\left(g^{i j}(x)\right)$ be positive definite everywhere. We may thus interpret the functions $g^{i j}(x)$ as the contravariant components of a Riemannian metric

$$
d s^{2}=\sum_{i, j=1}^{m} g_{i j}(x) d x^{i} d x^{j}
$$

where $\left(g_{i j}(x)\right)$ is the inverse of $g^{i j}(x)$. It is convenient to express $L$ in covariant form as follows:

$$
L=-\sum_{i, j=1}^{m} g^{i j}\left(\nabla_{i}-A_{i}\right)\left(\nabla_{j}-A_{j}\right)+V,
$$

where $\nabla_{i}$ is the covariant derivative associated to the metric (49), and

$$
\begin{aligned}
A_{j} & =\sum_{j=1}^{m} g_{i j}\left[\frac{b^{i}}{2}+\frac{1}{2 \sqrt{g}} \sum_{k=1}^{m} \frac{\partial}{\partial x^{k}}\left(\sqrt{g} g^{i k}\right)\right], \quad A^{i}=\sum_{j=1}^{m} g^{i j} A_{j}, \\
V & =c+\sum_{i=1}^{m}\left[A_{i} A^{i}-\frac{1}{\sqrt{g}} \frac{\partial}{\partial x^{i}}\left(\sqrt{g} A^{i}\right)\right],
\end{aligned}
$$

with $g=\operatorname{det}\left(g_{i j}\right)$. We define the magnetic one form associated with such an operator to be

$$
\omega=\sum_{i=1}^{m} A_{i} d x^{i}
$$

Theorem 14. The necessary and sufficient condition for an elliptic second order differential operator $L$ to be equivalent to a Schrödinger operator is that its magnetic one form be closed:

$$
d \omega=0 .
$$

For a given Lie algebra of differential operators $\mathfrak{g}$, Equation (50) is equivalent to a set of algebraic equations in the coefficients $C_{a b}$ and $C_{a}$, which are called the closure conditions. In the complex case, these conditions were extensively analyzed in [13], although their complete solution, and hence the complete classification of quasi-exactly solvable Schrödinger operators, remains problematic.

We now proceed to construct a few new examples of quasi-exactly solvable Hamiltonians based on the classification of the real Lie algebras of first-order differential operators developed in the previous section. According to Theorem 11, all the additional real canonical forms are subalgebras of Case 4.5, so that there is no loss of generality in working exclusively with $\mathfrak{s o}(3,1)$. Interestingly, although some of the additional real normal forms found in the previous section have already been used to obtain quasi-exactly solvable potentials - see, for instance, $[\mathbf{2 4}],[\mathbf{2 6}],[\mathbf{2 7}]$ for $\mathfrak{s o}(3)$ - we are not aware of any quasi-exactly solvable potential that has been explicitly linked to $\mathfrak{s o}(3,1)$ in the literature. However, the algebra $\mathfrak{s o}(3,1)$ does occur in the chain of subalgebras leading to the $\mathfrak{s o}(4,2)$ spectrum 
generating algebra for the hydrogen atom, arising from the conserved angular momentum and Runge-Lenz vectors; see the review article $[2]$. The only example of quasi-exactly solvable $\mathfrak{s o}(3,1)$ potential that we know of is the remarkable multiparameter family recently constructed by Zaslavskii, [31; eq. (31-33)]. Although the Hamiltonians in this family were obtained without explicitly using Lie-algebraic techniques, it can be shown (cf. [32]) that they arise from Hamiltonians that are quasi-exactly solvable with respect to the Lie algebra $\mathfrak{s l}(2) \oplus \mathfrak{s l}(2)$ of Type 1.4, when one performs the change of variables (16), and imposes that the resulting second-order differential operator have real coefficients. In fact, these Hamiltonians could have been obtained much more directly by starting with the most general Lie-algebraic second-order differential operator by taking an arbitrary polynomial of degree two in the generators of $\mathfrak{s o}(3,1)$, and imposing that $i)$ the coordinates for the induced metric be isothermal, and ii) the closure conditions be satisfied. It can be shown that the most general Hamiltonian satisfying these two conditions depends on 15 real parameters satisfying 9 algebraic constraints. Thus, the set of all such Hamiltonians is parametrized by a 6-dimensional algebraic variety. Although the number of essential parameters in Zaslavskii's multiparameter family is 6 , we shall now show that the latter family is only one of several components of the variety. Indeed, we now present a different 6-parameter family of Hamiltonians satisfying the above two conditions.

Indeed, consider the family of second-order differential operators (48) defined by the following choice of the coefficients $C_{a b}$ and $C_{a}$ :

$$
\left(C_{a b}\right)=\left(\begin{array}{cccccc}
\alpha & 0 & 0 & 0 & \mu / 2 & -\beta \\
0 & \alpha & 0 & 0 & 0 & 0 \\
0 & 0 & 0 & \beta & \gamma & \lambda \\
0 & 0 & \beta & \mu & -\lambda & \gamma \\
\mu / 2 & 0 & \gamma & -\lambda & \nu & 0 \\
-\beta & 0 & \lambda & \gamma & 0 & \nu
\end{array}\right), \quad\left(C_{a}\right)=-2 n(0,0,0, \beta, \gamma, \lambda)
$$

where $T^{1}, \ldots, T^{6}$ are the $\mathfrak{s o}(3,1)$ generators given by $(45)$. (We omit $T^{0}=1$ without loss of generality.) A long but straightforward calculation shows that the closure conditions are satisfied. The associated metric is

$$
d s^{2}=A^{-1}\left(d x^{2}+d y^{2}\right)
$$

with

$$
A=\alpha+\mu x^{2}-2 \beta x y+2 \gamma x\left(x^{2}+y^{2}\right)+2 \lambda y\left(x^{2}+y^{2}\right)+\nu\left(x^{2}+y^{2}\right)^{2},
$$

so that the $(x, y)$ coordinates are isothermal. The Gaussian curvature is given by

$\kappa=A^{-1}\left[a+b x+c y+d\left(x^{2}+y^{2}\right)+e x y+f x^{2}+g x\left(x^{2}+y^{2}\right)+h y\left(x^{2}+y^{2}\right)+k\left(x^{2}+y^{2}\right)^{2}\right]$,

with

$$
\begin{array}{lll}
a=\alpha \mu, & b=8 \alpha \gamma, & c=8 \alpha \lambda, \\
d=2\left(4 \alpha \nu-\beta^{2}\right), & e=2 \beta \mu, & f=-\mu^{2}, \\
g=2(2 \beta \lambda-\gamma \mu), & h=2(2 \beta \gamma+\lambda \mu), & k=\mu \nu-2 \gamma^{2}-2 \lambda^{2} .
\end{array}
$$


Since the closure conditions are satisfied, we know that $L$ is equivalent under a gauge transformation to a Schrödinger operator (47) on the open subset of $\mathbb{R}^{2}$ where $A$ is positive, with metric given by (51). In fact, we have

$$
H=\eta T \eta^{-1}=-\Delta+V(x, y), \quad \text { where } \quad \eta=A^{-n / 2},
$$

and the potential is

$$
\begin{aligned}
& V=\frac{n(n+2)}{A}\left[-4 \alpha(\gamma x+\lambda y)+x^{2} \mu^{2}-2 \beta \mu x y+\left(\beta^{2}-4 \alpha \nu\right)\left(x^{2}+y^{2}\right)\right. \\
& \left.\quad+2 x(\gamma \mu-\beta \lambda)\left(x^{2}+y^{2}\right)-2 \beta \gamma y\left(x^{2}+y^{2}\right)+\left(\gamma^{2}+\lambda^{2}\right)\left(x^{2}+y^{2}\right)^{2}\right] .
\end{aligned}
$$

It should be noted that for generic values of the parameters neither the above solution of the closure conditions (nor the one given by Zaslavskii), satisfy the additional condition that the associated metric be positive definite in all of $\mathbb{R}^{2}$.

One can obtain many other multiparameter families of $\mathfrak{s o}(3,1)$ potentials, for instance by dropping the condition that the $(x, y)$ coordinates be isothermal for the metric. We shall content ourselves with the following example, in which

$$
\left(C_{a b}\right)=\operatorname{diag}(\alpha, \alpha, \beta, \gamma, \lambda, \lambda), \quad\left(C_{a}\right)=0 .
$$

Again, the closure conditions are satisfied by the above choice of coefficients. The associated contravariant metric tensor $\left(g^{i j}\right)$ is given by

$$
\begin{aligned}
& g^{11}=\alpha+\beta x^{2}+\gamma y^{2}+\lambda\left(x^{2}+y^{2}\right)^{2}, \\
& g^{12}=(\beta-\gamma) x y, \\
& g^{22}=\alpha+\gamma x^{2}+\beta y^{2}+\lambda\left(x^{2}+y^{2}\right)^{2} .
\end{aligned}
$$

The Gaussian curvature is

$$
\kappa=\frac{(-\beta+3 \gamma)\left(\alpha^{2}+\lambda^{2} r^{8}\right)+2(\beta \gamma+4 \alpha \lambda) r^{2}\left(\alpha+\lambda r^{4}\right)+2 \alpha \lambda(5 \beta+\gamma) r^{4}}{\left(\alpha+\gamma r^{2}+\lambda r^{4}\right)^{2}}
$$

with $r^{2}=x^{2}+y^{2}$. In contrast with the previous case, if the parameters $\alpha, \beta, \gamma$ and $\lambda$ are positive, then the metric is non-singular and positive definite for $(x, y)$ ranging over all of $\mathbb{R}^{2}$.

As before, the fact that the closure conditions are satisfied guarantees the existence of a gauge factor $\eta$ such that $H=\eta L \eta^{-1}$ is a Schrödinger operator. If

$$
\rho=4 \alpha \lambda-\beta^{2},
$$

the gauge factor is given by 


$$
\eta= \begin{cases}\exp \left(\frac{n \beta}{\sqrt{\rho}} \arctan \frac{\beta+2 \lambda r^{2}}{\sqrt{\rho}}\right)\left(\alpha+\beta r^{2}+\lambda r^{4}\right)^{-\frac{1}{4}-\frac{n}{2}}\left(\alpha+\gamma r^{2}+\lambda r^{4}\right)^{\frac{1}{4}}, & \rho>0 \\ \exp \left(-\frac{2 n \alpha}{2 \alpha+\beta r^{2}}\right)\left(2 \alpha+\beta r^{2}\right)^{-\frac{1}{2}-n}\left(4 \alpha^{2}+4 \alpha \gamma r^{2}+\beta^{2} r^{4}\right)^{\frac{1}{4}}, & \rho=0 \\ \left(\alpha+\beta r^{2}+\lambda r^{4}\right)^{-\frac{3}{4}-n}\left(\alpha+\gamma r^{2}+\lambda r^{4}\right)^{\frac{1}{4}}\left(\frac{2 \lambda r^{2}+\beta-\sqrt{-\rho}}{2 \lambda r^{2}+\beta+\sqrt{-\rho}}\right)^{\frac{n \beta}{\sqrt{-\rho}}}, & \rho<0 .\end{cases}
$$

In all cases, the expression for the potential $V$ is

$$
\begin{gathered}
4 V=\frac{16 \alpha \beta n(1+n)+r^{2}\left[\beta^{2}\left(3+16 n+16 n^{2}\right)-4 \alpha \lambda\left(3+8 n+4 n^{2}\right)\right]}{\alpha+\beta r^{2}+\lambda r^{4}} \\
+\frac{5(\beta-\gamma)\left(4 \alpha \lambda-\gamma^{2}\right)+3 \lambda\left(2 \beta \gamma-3 \gamma^{2}+4 \alpha \lambda\right) r^{2}}{\lambda\left(\alpha+\gamma r^{2}+\lambda r^{4}\right)} \\
-\frac{5(\beta-\gamma)\left(4 \alpha \lambda-\gamma^{2}\right)\left(\alpha+\gamma r^{2}\right)}{\lambda\left(\alpha+\gamma r^{2}+\lambda r^{4}\right)^{2}}+4 \gamma-4 \beta(1+2 n)^{2},
\end{gathered}
$$

with $r^{2}=x^{2}+y^{2}$. Since the potential is a function of $r$ only, it is natural to look for eigenfunctions of $H$ which depend on $r$ only. When this is done, it can be shown that one ends up with an effective Hamiltonian on the line which is an element of the enveloping algebra of the standard realization of $\mathfrak{s l}(2, \mathbb{R})$ in one dimension. Thus, no new quasi-exactly solvable one-dimensional potentials are obtained by reduction of the above quasi-exactly solvable $\mathfrak{s o}(3,1)$ potential. This lends additional support to the observation of $[\mathbf{1 3}]$ that reduction of two-dimensional quasi-exactly solvable Schrödinger operators does not lead to any new one-dimensional quasi-exactly solvable operators. However, a full explanation of this fact remains obscure.

Acknowledgment: It is a pleasure to thank the referees for useful comments. 


\section{References}

[1] Ackerman, M., and Hermann, R., Sophus Lie's 1880 Transformation Group Paper, Math Sci Press, Brookline, Mass., 1975.

[2] Adams, B.G., Čižek, J., and Paldus, J., Lie algebraic methods and their applications to simple quantum systems, Adv. Quantum Chem. 19 (1988), 1-85.

[3] Barut, A.O., and Böhm, A., Dynamical groups and mass formula, Phys. Rev. 139 (1965), B1107-B1112.

[4] Bohm, A., and Ne'eman, Y., Dynamical groups and spectrum generating algebras, in: Dynamical Groups and Spectrum Generating Algebras, Bohm, A., Ne'eman, Y., Barut, A.O., eds., World Scientific, Singapore, 1988, pp. 3-68.

[5] Bohm, A., Ne'eman, Y., and Barut, A.O. (eds.), Dynamical Groups and Spectrum Generating Algebras, World Scientific, Singapore, 1988.

[6] Dothan, Y., Gell-Mann, M., and Ne'eman, Y., Series of hadron energy levels as representations of non-compact groups, Phys. Lett. 17 (1965), 148-151.

[7] González-López, A., Hurtubise, J., Kamran, N., and Olver, P.J., Quantification de la cohomologie des algèbres de Lie de champs de vecteurs et fibrés en droites sur des surfaces complexes compactes, Comptes Rendus Acad. Sci. (Paris), Série I, 316 (1993), 1307-1312.

[8] González-López, A., Kamran, N., and Olver, P.J., Quasi-exactly solvable Lie algebras of first order differential operators in two complex variables, J. Phys. A 24 (1991), 3995-4008.

[9] González-López, A., Kamran, N., and Olver, P.J., Lie algebras of first order differential operators in two complex variables, in: Differential Geometry, Global Analysis, and Topology, A. Nicas and W.F. Shadwick, eds., Canadian Math.

Soc. Conference Proceedings, vol. 12, Amer. Math. Soc., Providence, R.I., 1991, pp. 51-84.

[10] González-López, A., Kamran, N., and Olver, P.J., Lie algebras of differential operators in two complex variables, American J. Math. 114 (1992), 1163-1185.

[11] González-López, A., Kamran, N., and Olver, P.J., Lie algebras of vector fields in the real plane, Proc. London Math. Soc. 64 (1992), 339-368.

[12] González-López, A., Kamran, N., and Olver, P.J., Normalizability of one-dimensional quasi-exactly solvable Schrödinger operators, Commun. Math. Phys. 153 (1993), 117-146.

[13] González-López, A., Kamran, N., and Olver, P.J., New quasi-exactly solvable Hamiltonians in two dimensions, Commun. Math. Phys. 159 (1994), 503-537.

[14] González-López, A., Kamran, N., and Olver, P.J., Quasi-exact solvability, Contemp. Math. 160 (1994), 113-140.

[15] Goshen, S., and Lipkin, H.J., A simple independent-particle system having collective properties, Ann. Phys. 6 (1959), 301-309.

[16] Gruber, B., and Otsuka, T. (eds.), Symmetries in Science VII: Spectrum-Generating Algebras and Dynamic Symmetries in Physics, Plenum Press, New York, 1993.

[17] Jacobson, N., Lie Algebras, Interscience Publ. Inc., New York, 1962. 
[18] Iachello, F., and Levine, R.D., Algebraic Theory of Molecules, Oxford Univ. Press, Oxford, 1995.

[19] Kamran, N., and Olver, P.J., Lie algebras of differential operators and Lie-algebraic potentials, J. Math. Anal. Appl. 145 (1990), 342-356.

[20] Lie, S., Theorie der Transformationsgruppen, vol. 3, B.G. Teubner, Leipzig, 1893.

[21] Lie, S., Gruppenregister, in: Gesammelte Abhandlungen, vol. 5, B.G. Teubner, Leipzig, 1924, pp. 767-773.

[22] Lie, S., Theorie der Transformationsgruppen, in: Gesammelte Abhandlungen, Vol. 6, B.G. Teubner, Leipzig, 1927, pp. 1-94; see [1] for an English translation.

[23] Miller, W., Jr., Lie Theory and Special Functions, Academic Press, New York, 1968.

[24] Morozov, A.Y., Perelomov, A.M., Rosly, A.A., Shifman, M.A., and Turbiner, A.V., Quasi-exactly solvable quantal problems: one-dimensional analogue of rational conformal field theories, Int. J. Mod. Phys. A 5 (1990), 803-832.

[25] Olver, P.J., Equivalence, Invariants, and Symmetry, Cambridge University Press, 1995.

[26] Shifman, M.A., New findings in quantum mechanics (partial algebraization of the spectral problem), Int. J. Mod. Phys. A 126 (1989), 2897-2952.

[27] Shifman, M.A., and Turbiner, A.V., Quantal problems with partial algebraization of the spectrum, Commun. Math. Phys. 126 (1989), 347-365.

[28] Turbiner, A.V., Quasi-exactly solvable problems and $\mathfrak{s l}(2)$ algebra, Commun. Math. Phys. 118 (1988), 467-474.

[29] Ushveridze, A. G., Quasi-exactly solvable models in quantum mechanics, Sov. J. Part. Nucl. 20 (1989), 504-528.

[30] Ushveridze, A. G., Quasi-exactly Solvable Models in Quantum Mechanics, Inst. of Physics Publ., Bristol, England, 1994.

[31] Zaslavskii, O.B., Quasi-exactly solvable models from finite-dimensional matrices, $J$. Phys. A 26 (1993), 6563-6574.

[32] Zaslavskii, O.B., Two-dimensional quasi-exactly solvable models and classical orthogonal polynomials, J. Phys. A 27 (1994), L323-328. 
Table 1

Transitive, Imprimitive Lie Algebras of Vector Fields in $\mathbb{C}^{2}$

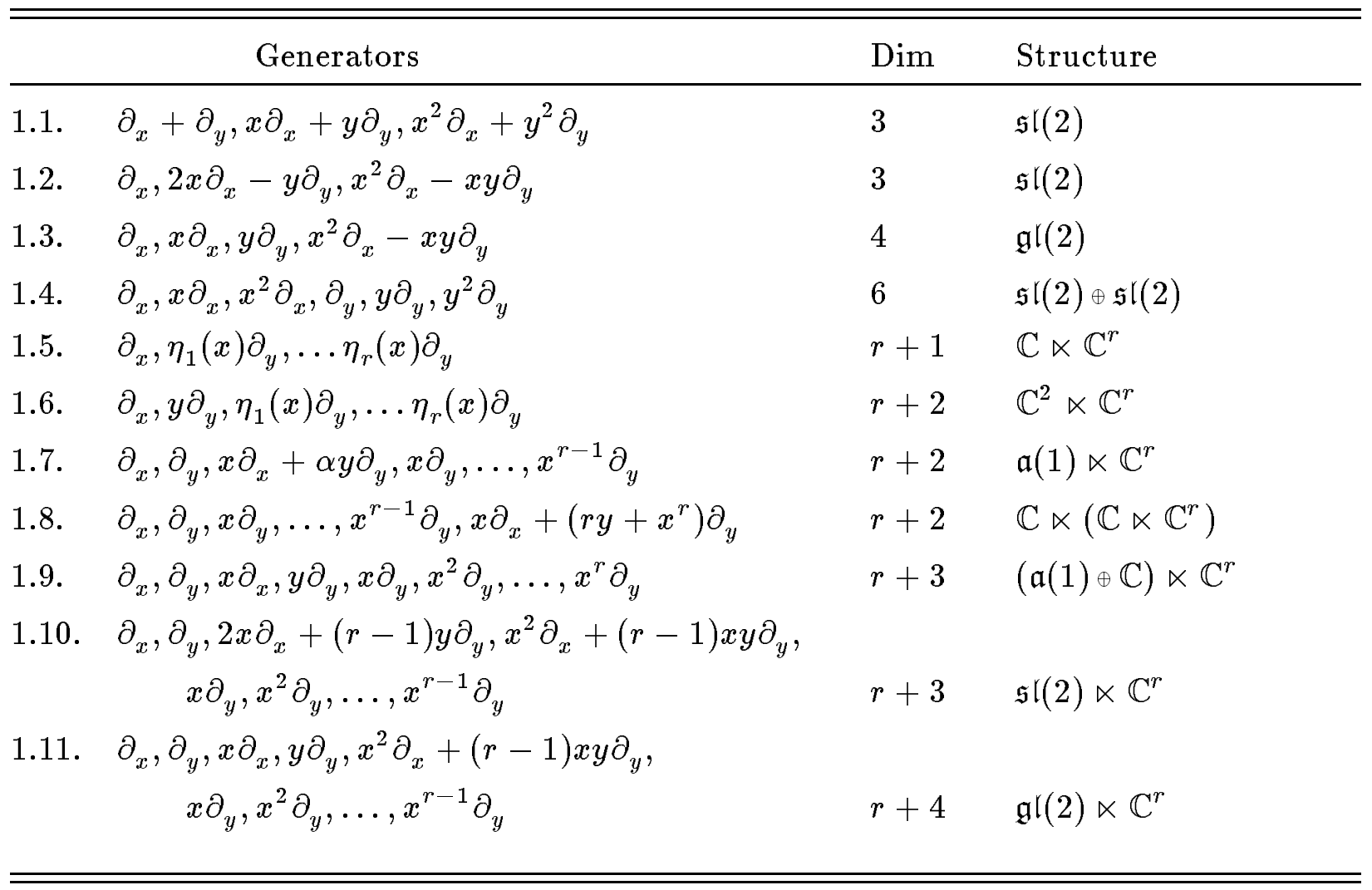

In Cases 1.5 and 1.6, the functions $\eta_{1}(x), \ldots, \eta_{r}(x)$ satisfy an $r^{\text {th }}$ order constant coefficient homogeneous linear ordinary differential equation $\mathcal{D}[u]=0$. In Cases $1.5-1.11$ we require $r \geq 1$. Note, though, that if we set $r=0$ in Case 1.10 , and replace $y$ by $y^{2}$, we obtain Case 1.1. Similarly, if we set $r=0$ in Case 1.11, we obtain Case 1.3. Cases 1.7 and 1.8 for $r=0$ are equivalent to the Lie algebra $\left\{\partial_{x}, e^{x} \partial_{y}\right\}$ of type 1.5 . Case 1.9 for $r=0$ is equivalent to the Lie algebra $\left\{\partial_{x}, \partial_{y}, y \partial_{y}\right\}$ of type 1.6. Finally, Case 1.7 for $\alpha=0, r=1$ is the translation algebra, which also appears in Case 1.6 for $r=1, \eta_{1}=1$.

Note: We use $\mathfrak{a}(n)$ to denote the Lie algebra of the affine group of $\mathbb{C}^{n}$ (or, in the real case, $\left.\mathbb{R}^{n}\right)$, and $\mathfrak{s a}(n)$ for the Lie algebra of the special affine group consisting of volumepreserving affine transformations. 
Table 2

Primitive Lie Algebras of Vector Fields in $\mathbb{C}^{2}$

\begin{tabular}{llll}
\hline \hline & Generators & Dim & Structure \\
\hline 2.1. & $\partial_{x}, \partial_{y}, x \partial_{x}-y \partial_{y}, y \partial_{x}, x \partial_{y}$ & 5 & $\mathfrak{s a}(2)$ \\
2.2. & $\partial_{x}, \partial_{y}, x \partial_{x}, y \partial_{x}, x \partial_{y}, y \partial_{y}$ & 6 & $\mathfrak{a}(2)$ \\
2.3. & $\partial_{x}, \partial_{y}, x \partial_{x}, y \partial_{x}, x \partial_{y}, y \partial_{y}, x^{2} \partial_{x}+x y \partial_{y}, x y \partial_{x}+y^{2} \partial_{y}$ & 8 & $\mathfrak{s l}(3)$ \\
\hline \hline
\end{tabular}

Table 3

Intransitive Lie Algebras of Vector Fields in $\mathbb{C}^{2}$

\begin{tabular}{llll}
\hline \hline & \multicolumn{1}{c}{ Generators } & Dim & Structure \\
\hline 3.1. & $\eta_{1}(x) \partial_{y}, \ldots, \eta_{r}(x) \partial_{y}$ & $r$ & $\mathbb{C}^{r}$ \\
3.2. & $\eta_{1}(x) \partial_{y}, \ldots, \eta_{r}(x) \partial_{y}, y \partial_{y}$ & $r+1$ & $\mathbb{C} \ltimes \mathbb{C}^{r}$ \\
3.3. & $\partial_{x}, x \partial_{x}, x^{2} \partial_{x}$ & 3 & $\mathfrak{s l}(2)$ \\
& & & \\
\hline \hline
\end{tabular}

In Cases 3.1 and $3.2, r \geq 1$, and the functions $\eta_{1}(x), \ldots, \eta_{r}(x)$ are arbitrary.

Table 4

Additional Primitive Lie Algebras of Vector Fields in $\mathbb{R}^{2}$

\begin{tabular}{|c|c|c|c|}
\hline Generators & Dim & Structure & Complex Form \\
\hline 4.1. $\quad \partial_{x}, x \partial_{x}+y \partial_{y},\left(x^{2}-y^{2}\right) \partial_{x}+2 x y \partial_{y}$ & 3 & $\mathfrak{s l}(2)$ & 1.1 \\
\hline \multicolumn{4}{|l|}{ 4.2. $y \partial_{x}-x \partial_{y},\left(1+x^{2}-y^{2}\right) \partial_{x}+2 x y \partial_{y}$} \\
\hline $2 x y \partial_{x}+\left(1-x^{2}+y^{2}\right) \partial_{y}$ & 3 & $\mathfrak{s o}(3)$ & 1.1 \\
\hline 4.3. $\partial_{x}, \partial_{y}, \beta\left(x \partial_{x}+y \partial_{y}\right)+y \partial_{x}-x \partial_{y}$ & 3 & $\mathbb{R} \ltimes \mathbb{R}^{2}$ & $1.7(r=1)$ \\
\hline 4.4. $\quad \partial_{x}, \partial_{y}, x \partial_{x}+y \partial_{y}, y \partial_{x}-x \partial_{y}$ & 4 & $\mathbb{R}^{2} \ltimes \mathbb{R}^{2}$ & $1.9(r=1)$ \\
\hline$\left(x^{2}-y^{2}\right) \partial_{x}+2 x y \partial_{y}, 2 x y \partial_{x}+\left(y^{2}-x^{2}\right) \partial_{y}$ & 6 & $\mathfrak{s o}(3,1)$ & 1.4 \\
\hline
\end{tabular}


Table 5

Finite-dimensional Modules for Lie Algebras of Vector Fields in $\mathbb{R}^{2}$

\begin{tabular}{|c|c|c|c|}
\hline \multicolumn{2}{|c|}{ Monomials? } & Generators & Rules \\
\hline 1.1 & No & $(x-y)^{n-k} R_{k}^{n}\left(\frac{x+y}{x-y}\right)$ & $0 \leq k \leq 2 n$ \\
\hline 1.2 & Yes & $x^{j} y^{n}$ & $0 \leq j \leq n$ \\
\hline 1.3 & Yes & $x^{j} y^{n}$ & $0 \leq j \leq n$ \\
\hline 1.4 & Yes & 1 & \\
\hline 1.5 & No & $x^{j} y^{k} e^{\mu x} e^{\nu y}$ & $(j, k, \mu, \nu) \longrightarrow(j-1, k, \mu, \nu),\left(j+r_{\lambda}, k-1, \mu+\lambda, \nu\right)$ \\
\hline 1.6 & Yes & $x^{j} y^{k} e^{\mu x}$ & $(j, k, \mu) \longrightarrow(j-1, k, \mu),\left(j+r_{\lambda}, k-1, \mu+\lambda\right)$ \\
\hline 1.7 & $r-1$ & $<\alpha \in \mathbb{Q}^{+} \quad x^{j} y^{k}$ & $(j, k) \longrightarrow(j-1, k),(j+r-1, k-1)$ \\
\hline 1.8 & Yes & $x^{j} y^{k}$ & $(j, k) \longrightarrow(j-1, k),(j+r, k-1)$ \\
\hline 1.9 & Yes & $x^{j} y^{k}$ & $(j, k) \longrightarrow(j-1, k),(j+r, k-1)$ \\
\hline 1.10 & Yes & $y^{k} e^{\nu y}$ & $0 \leq k \leq n_{\nu} \quad\left(\nu=0, n_{\nu}=0\right.$ when $\left.r>1\right)$ \\
\hline 1.11 & Yes & $y^{k}$ & $0 \leq k \leq n \quad(n=0$ when $r>1)$ \\
\hline 2.1 & Yes & $x^{j} y^{k}$ & $0 \leq j+k \leq n$ \\
\hline 2.2 & Yes & $x^{j} y^{k}$ & $0 \leq j+k \leq n$ \\
\hline 2.3 & Yes & 1 & \\
\hline 3.1 & No & $g(x) y^{k} e^{\nu y}$ & $(k, \nu, g) \longrightarrow\left(k-1, \nu, g \cdot \eta_{i}\right)$ \\
\hline 3.2 & Yes & $y^{k} g(x)$ & $(k, g) \longrightarrow\left(k-1, g \cdot \eta_{i}\right)$ \\
\hline 3.3 & Yes & $g(y)$ & \\
\hline 4.1 & No & $y^{n-k} R_{k}^{n}\left(\frac{i x}{y}\right)$ & $0 \leq k \leq 2 n$ \\
\hline 4.2 & No & $\left(\frac{x^{2}+y^{2}+1}{x-i y}\right)^{n-k} R_{k}^{n}$ & $\left(\frac{x^{2}+y^{2}-1}{x^{2}+y^{2}+1}\right)$ \\
\hline 4.3 & No & $\left(x^{2}+y^{2}\right)^{j} P_{k}(x, y)$ & $P_{k} \in \mathcal{H}^{(k)}, \quad 0 \leq k \leq m-j, \quad 0 \leq j \leq n \leq m$ \\
\hline 4.4 & No & $\left(x^{2}+y^{2}\right)^{j} P_{k}(x, y)$ & $P_{k} \in \mathcal{H}^{(k)}, \quad 0 \leq k \leq m-j, \quad 0 \leq j \leq n \leq m$ \\
\hline 4.5 & Yes & 1 & \\
\hline
\end{tabular}

Here $m, n$, and $n_{\nu}$ are nonnegative integers. Further, $\mathcal{H}^{(k)}$ denotes the space of harmonic polynomials of degree at most $k$. In Case $1.5, \nu=0$ unless $\mathfrak{h}$ is the translation algebra $\operatorname{Span}\left\{\partial_{x}, \partial_{y}\right\}$. In cases 1.5 and $1.6, \lambda$ is a root of the characteristic polynomial of $\mathcal{D}$ with algebraic multiplicity $r_{\lambda}+1$. Finally, $R_{k}^{n}(t)=\frac{d^{k}}{d t^{k}}\left(t^{2}-1\right)^{n}$. 
Table 6

Cohomology for Lie Algebras of Vector Fields in $\mathbb{R}^{2}$

\begin{tabular}{|c|c|c|}
\hline \multicolumn{2}{|r|}{ Dimension } & Representatives \\
\hline 1.1 & 1 & $x^{2} \partial_{x}+y^{2} \partial_{y}+c_{1}(x-y)$ \\
\hline 1.2 & 1 & $x^{2} \partial_{x}-x y \partial_{y}+c_{1} y^{-2}$ \\
\hline 1.3 & $0(1 \in \mathfrak{M}), 1(1 \notin \mathfrak{M})$ & $x \partial_{x}+c_{1}, \quad y \partial_{y}+2 c_{1}$ \\
\hline 1.4 & 2 & $x \partial_{x}+c_{1}, x^{2} \partial_{x}+2 c_{1} x, y \partial_{y}+c_{2}, y^{2} \partial_{y}+2 c_{2} y$ \\
\hline 1.5 & $<\infty$ & $\eta_{k}(x) \partial_{y}+\zeta_{k}(x, y)$ \\
\hline 1.6 & 1 & $y \partial_{y}+c_{1} x^{n}$ \\
\hline 1.7 & $0(\alpha \notin \mathbb{Q}), 1$ or $0(\alpha \in \mathbb{Q})$ & $x \partial_{x}+\alpha y \partial_{y}+c_{1} x^{i} y^{j}$, or $\eta_{k}(x) \partial_{y}+c_{k} x^{i+k} y^{j}$ \\
\hline 1.8 & $0(\mathfrak{M} \neq 0), 1(\mathfrak{M}=0)$ & $x \partial_{x}+\left(r y+x^{r} y\right) \partial_{y}+c_{1}$ \\
\hline 1.9 & $0(\mathfrak{M} \neq 0), 2(\mathfrak{M}=0)$ & $x \partial_{x}+c_{1}, \quad y \partial_{y}+c_{2}$ \\
\hline 1.10 & $1(r \neq 3)$ & $2 x \partial_{x}+(r-1) y \partial_{y}+c_{1}, \quad x^{2} \partial_{x}+(r-1) x y \partial_{y}+c_{1} x$ \\
\hline & $2(r=3) \quad x \partial_{x}+y \partial_{y}+$ & $c_{1}, x \partial_{y}+c_{2}, x^{2} \partial_{x}+2 x y \partial_{y}+2 c_{1} x+2 c_{2} y, x^{2} \partial_{y}+2 c_{2} x$ \\
\hline 1.11 & $1(\mathfrak{M} \neq 0), 2(\mathfrak{M}=0) x \partial_{x}$ & $+c_{1}, y \partial_{y}+c_{2}, x^{2} \partial_{x}+(r-1) x y \partial_{y}+\left(2 c_{1}+(r-1) c_{2}\right) x$ \\
\hline 2.1 & $0(\mathfrak{M} \neq \mathbb{R}), 1(\mathfrak{M}=\mathbb{R})$ & $\partial_{y}+2 c_{1} x, \quad y \partial_{x}+c_{1} y^{2}, \quad x \partial_{y}+c_{1} x^{2}$ \\
\hline 2.2 & $0(\mathfrak{M} \neq 0), 1(\mathfrak{M}=0)$ & $x \partial_{x}+c_{1}, \quad y \partial_{y}+c_{1}$ \\
\hline 2.3 & 1 & $c_{1}, x^{2} \partial_{x}+x y \partial_{y}+3 c_{1} x, y \partial_{y}+c_{1}, x y \partial_{x}+y^{2} \partial_{y}+3 c_{1} y$ \\
\hline 3.1 & $0(r=1), \infty(r>1)$ & $\eta_{i}(x) \partial_{y}+f_{i}(x) y^{j}$ \\
\hline 3.2 & $\infty$ & $y \partial_{y}+f(x)$ \\
\hline 3.3 & $\infty$ & $x \partial_{x}+h(y), \quad x^{2} \partial_{x}+2 x h(y)$ \\
\hline 4.1 & 1 & $\left(x^{2}-y^{2}\right) \partial_{x}+2 x y \partial_{y}+c_{1} y$ \\
\hline 4.2 & $\left(1+x^{2}\right.$ & $\left.y^{2}\right) \partial_{x}+2 x y \partial_{y}+c_{1} y, 2 x y \partial_{x}+\left(1-x^{2}+y^{2}\right) \partial_{y}-c_{1} x$ \\
\hline 4.3 & $0(\beta \neq 0), 1(\beta=0)$ & $\begin{cases}\partial_{x}+c_{1} y\left(x^{2}+y^{2}\right)^{m}, \partial_{y}-c_{1} x\left(x^{2}+y^{2}\right)^{m}, & n=2 m \\
y \partial_{x}-x \partial_{y}+c_{1}\left(x^{2}+y^{2}\right)^{m+1}, & n=2 m+1\end{cases}$ \\
\hline 4.4 & $0(\mathfrak{M} \neq 0), 2(\mathfrak{M}=0)$ & $x \partial_{x}+y \partial_{y}+c_{1}, x \partial_{y}-y \partial_{x}+c_{2}$ \\
\hline 4.5 & 2 & $\left\{\begin{array}{l}\left(x^{2}-y^{2}\right) \partial_{x}+2 x y \partial_{y}+c_{1} x+c_{2} y \\
2 x y \partial_{x}+\left(y^{2}-x^{2}\right) \partial_{y}-c_{2} x+c_{1} y\end{array}\right.$ \\
\hline
\end{tabular}

See $[\mathbf{9}],[\mathbf{1 0}]$, for details, particularly for Cases 1.5, 1.7 and 3.2. 
Table 7

Quasi-Exactly Solvable Lie Algebras of Differential Operators in $\mathbb{R}^{2}$

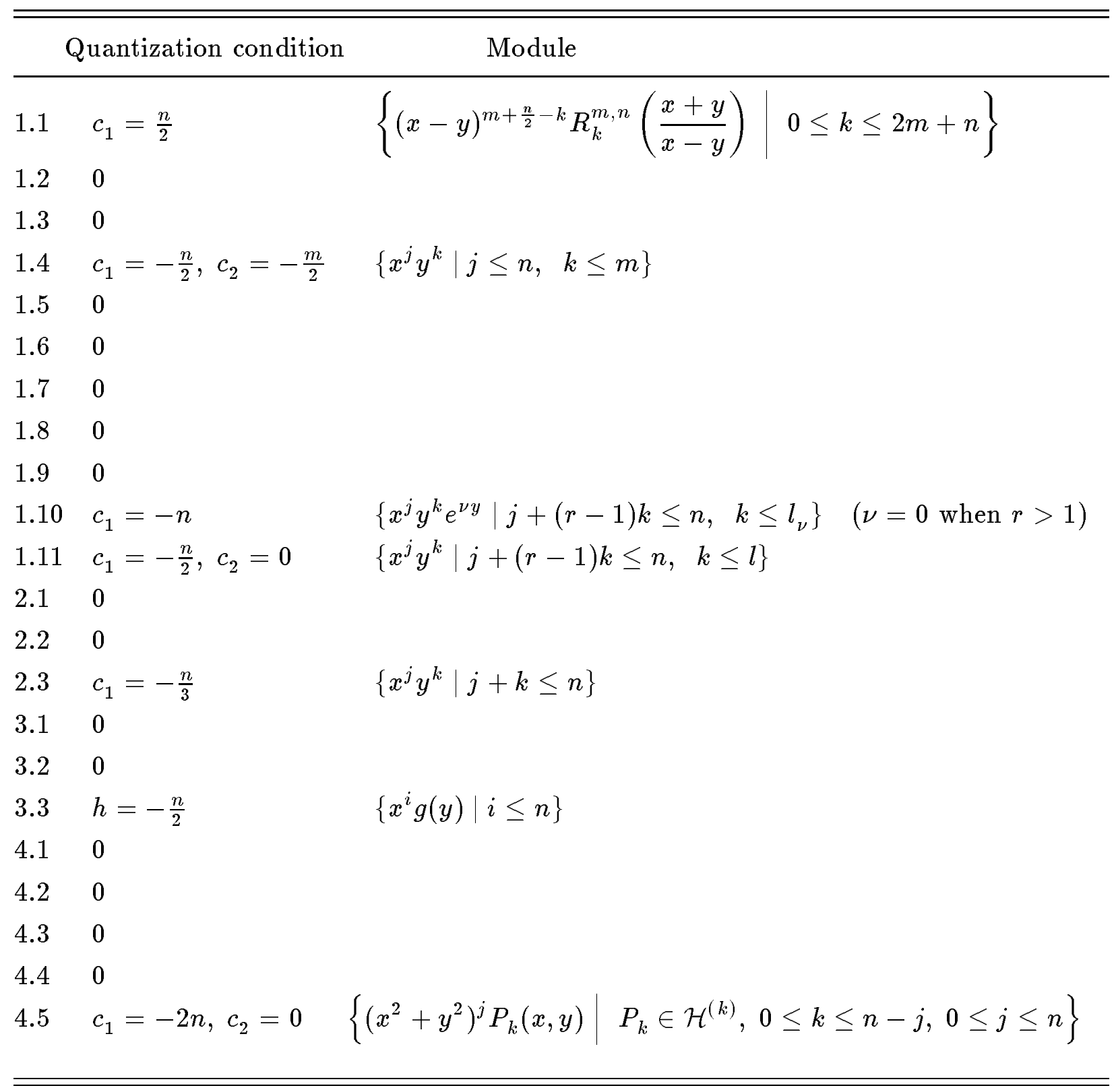

In this table, $l, l_{\nu}, m$, and $n$, are nonnegative integers, and

$$
R_{k}^{m, n}(t)=\frac{d^{k}}{d t^{k}}(t-1)^{m+n}(t+1)^{m}
$$

Recall, finally, that when the cohomology of $\mathfrak{g}$ vanishes the finite-dimensional $\mathfrak{g}$-modules are the $\mathfrak{h}$-modules listed in Table 5 . 